\title{
Joint Source-Channel Vector Quantization for Compressed Sensing
}

\author{
Amirpasha Shirazinia, Student Member, IEEE, Saikat Chatterjee, Member, IEEE, Mikael Skoglund, Senior Member,
} IEEE

\begin{abstract}
We study joint source-channel coding (JSCC) of compressed sensing (CS) measurements using vector quantizer (VQ). We develop a framework for realizing optimum JSCC schemes that enable encoding and transmitting CS measurements of a sparse source over discrete memoryless channels, and decoding the sparse source signal. For this purpose, the optimal design of encoder-decoder pair of a VQ is considered, where the optimality is addressed by minimizing end-to-end mean square error (MSE). We derive a theoretical lower-bound on the MSE performance, and propose a practical encoder-decoder design through an iterative algorithm. The resulting coding scheme is referred to as channeloptimized VQ for CS, coined COVQ-CS. In order to address the encoding complexity issue of the COVQ-CS, we propose to use a structured quantizer, namely low complexity multi-stage VQ (MSVQ). We derive new encoding and decoding conditions for the MSVQ, and then propose a practical encoder-decoder design algorithm referred to as channel-optimized MSVQ for CS, coined COMSVQ-CS. Through simulation studies, we compare the proposed schemes vis-a-vis relevant quantizers.
\end{abstract}

Index Terms-Vector quantization, multi-stage vector quantization, joint source-channel coding, noisy channel, compressed sensing, sparsity, mean square error.

\section{INTRODUCTION}

Compressed sensing (CS) [2] considers retrieving a highdimensional sparse vector $\mathbf{X}$ from relatively lower number of measurements. In many practical applications, the collected measurements at a CS sensor node need to be encoded using finite bits and transmitted over noisy communication channels. To do so, efficient design of source and channel codes should be considered for reliable transmission of the CS measurements over noisy channels. The optimum performance theoretically attainable in a point-to-point memoryless channel can be achieved using separate design of source and channel codes, but this performance requires infinite source and channel code block lengths resulting in delay as well as coding complexity. Considering finite-length sparse source and CS measurement vector, it is theoretically guaranteed that joint source-channel coding (JSCC) can provide better performance than a separate design of source and channel codes. Therefore, to design a practical coding method, we focus on optimal JSCC principles for CS in the current work. Denoting the reconstruction vector by $\widehat{\mathbf{X}}$ at a decoder, our main objective is to develop a generic framework for optimum JSCC of CS measurements using vector quantization, or in other words, optimum joint source-channel vector quantization for $C S$, such that $\mathbb{E}\left[\|\mathbf{X}-\widehat{\mathbf{X}}\|_{2}^{2}\right]$ is minimized.

\section{A. Background}

Recently, significant research interest has been devoted to design and analysis of source coding, e.g. quantization, for CS, and a wide range of problems has been formulated. Existing work on this topic is mainly divided into three categories.

1) The first category considers optimum quantizer design for quantization of CS measurements, where a CS reconstruction algorithm is held fixed at the decoder. Examples include [3] and [4], where CS reconstruction algorithms are LASSO and message passing, respectively. Based on analysis-by-synthesis principle, we have recently developed a quantizer design method in [5], where any CS reconstruction algorithm can be used.

2) The second category considers the design of a good CS reconstruction algorithm, where the quantizer is held fixed. CS reconstruction from noisy measurements - where the noise properties follow the effect of quantization - falls in the category. Examples are [6]-[15]. To elaborate, let us consider [9] where CS measurements are uniformly quantized and a convex optimization-based CS reconstruction algorithm, called basis pursuit dequantizing (BPDQ), is developed to suit the effect of uniform quantization. Further, the design of CS reconstruction algorithms and their performance bounds for reconstructing a sparse source from 1-bit quantized measurements have been investigated in [12]-[15].

3) Another line of previous work focuses on trade-offs between the quantization resources (e.g., quantization rate) and CS resources (e.g., number of measurements or complexity of CS reconstruction) [8], [16]-[18]. For example, in [18], a trade-off between number of measurements and quantization rate was established by introducing the concept of two compression regimes as quantification of resources - quantization compression regime and CS compression regime.

We mention that all the above works are dedicated to pure source coding through quantization of CS measurements. To the best of our knowledge, there is limited work on JSCC of CS measurements using vector quantizer (VQ). In this regard, we had our previous effort in [1]. The current paper is build upon the work of [1], and provides a comprehensive framework for developing optimum JSCC schemes to encode and transmit CS measurements (of a sparse source $\mathbf{X}$ ) over discrete memoryless channels, and to decode the sparse source so as to provide the reconstruction $\widehat{\mathbf{X}}$. The optimality is addressed by minimizing the MSE performance measure $\mathbb{E}\left[\|\mathbf{X}-\widehat{\mathbf{X}}\|_{2}^{2}\right]$.

\section{B. Contributions}

We first consider the optimal design of VQ encoder-decoder pair for CS in the sense of minimizing the MSE. Here, we 
stress that we use the VQ in its generic form. This is different from the design methods using uniform quantization [9] or 1-bit quantization of CS measurements [12]-[15]. Our contributions include

- Establishing (necessary) optimal encoding and decoding conditions for VQ.

- Providing a theoretical bound on the MSE performance.

- Developing a practical VQ encoder-decoder design through an iterative algorithm.

- Addressing the encoding complexity issue of VQ using a structured quantizer, namely low complexity multistage VQ (MSVQ), where we derive new encoder-decoder conditions for sub-optimal design of the MSVQ.

Our practical encoder-decoder designs consider ChannelOptimized VQ for CS, coined COVQ-CS, and ChannelOptimized MSVQ for CS, coined COMSVQ-CS. To demonstrate the strength of the proposed designs, we compare them with relevant quantizer design methods through different simulation studies. Particularly, we show that in noisy channel scenarios, the proposed COVQ-CS and COMSVQ-CS schemes provide better and more robust (against channel noise) performances compared to existing quantizers for CS followed by separate channel coding.

\section{Outline}

The rest of the paper is organized as follows. In Section $\Pi$ we introduce some preliminaries of CS. The optimal design and performance analysis of a joint source-channel VQ for CS are presented in Section III. In Section [V] we propose a practical VQ encoder-decoder design algorithm. Further, in Section $\mathrm{V}$, we deal with complexity issue by proposing the design of computationally- and memory-efficient MSVQ for CS. The performance comparison of the proposed quantization schemes with other relevant methods are made in Section VI, and conclusions are drawn in Section VII

\section{Notations}

Notations: Random variables (RV's) will be denoted by upper-case letters while their realizations (instants) will be denoted by the respective lower-case letters. Random vectors of dimension $n$ will be represented by boldface characters. We will denote a sequence of RV's $J_{1}, \ldots, J_{N}$ by $\mathbf{J}_{1}^{N}$; further, $\mathbf{J}_{1}^{N}=\mathbf{j}_{1}^{N}$ implies that $J_{1}=j_{1}, \ldots, J_{N}=j_{N}$. Matrices will be denoted by capital Greek letters, except that the square identity matrix of dimension $n$ is denoted by $\mathbf{I}_{n}$. The matrix operators determinant, trace, transpose and the maximum eigenvalue of a matrix are denoted by $\operatorname{det}(\cdot), \operatorname{Tr}(\cdot),(\cdot)^{\top}$, and $\lambda_{\max }(\cdot)$, respectively. Further, cardinality of a set is shown by $|\cdot|$. We will use $\mathbb{E}[\cdot]$ to denote the expectation operator. The $\ell_{p}$-norm $(p>0)$ of a vector $\mathbf{z}$ will be denoted by $\|\mathbf{z}\|_{p}=\left(\sum_{n=1}^{N}\left|z_{n}\right|^{p}\right)^{1 / p}$. Also, $\|\mathbf{z}\|_{0}$ represents $\ell_{0}$-norm which is the number of nonzero coefficients in $\mathbf{z}$.

\section{PRELIMINARIES OF CS}

In CS, a random sparse vector (where most coefficients are likely zero) $\mathbf{X} \in \mathbb{R}^{N}$ is linearly measured by a known sensing

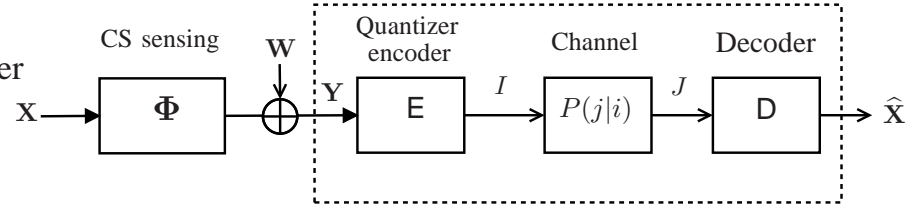

Fig. 1. Studied system model for joint source-channel vector quantization of CS measurements. The goal is to design encoder and decoder mappings (illustrated in dashed box) with respect to minimizing $\mathbb{E}\left[\|\mathbf{X}-\widehat{\mathbf{X}}\|_{2}^{2}\right]$ while the CS sensing matrix $\boldsymbol{\Phi}$ and channel $P(j \mid i)$ are known in advance.

matrix $\Phi \in \mathbb{R}^{M \times N}(M<N)$ resulting in an under-determined set of linear measurements (possibly) perturbed by noise

$$
\mathbf{Y}=\mathbf{\Phi} \mathbf{X}+\mathbf{W}
$$

where $\mathbf{Y} \in \mathbb{R}^{M}$ and $\mathbf{W} \in \mathbb{R}^{M}$ denote the measurement and the additive measurement noise vectors, respectively. We assume that $\mathbf{X}$ is a $K$-sparse vector, i.e., it has at most $K(K \leq M)$ nonzero coefficients, where the location and magnitude of the nonzero components are drawn from known distributions. We also assume that the sparsity level $K$ is known in advance. We define the support set of the sparse vector $\mathbf{X}=\left[X_{1}, \ldots, X_{N}\right]^{\top}$ as $\mathcal{S} \triangleq\left\{n: X_{n} \neq 0\right\} \subset\{1, \ldots, N\}$ with $|\mathcal{S}|=\|\mathbf{X}\|_{0} \leq K$. Next, we define the mutual coherence notion which characterizes the merit of a sensing matrix $\boldsymbol{\Phi}$. The mutual coherence is defined as [19]

$$
\mu \triangleq \max _{i \neq j} \frac{\left|\boldsymbol{\Phi}_{i}^{\top} \boldsymbol{\Phi}_{j}\right|}{\left\|\boldsymbol{\Phi}_{i}\right\|_{2}\left\|\boldsymbol{\Phi}_{j}\right\|_{2}}, \quad 1 \leq i, j \leq N,
$$

where $\boldsymbol{\Phi}_{i}$ denotes the $i^{\text {th }}$ column of $\boldsymbol{\Phi}$. The mutual coherence $0 \leq \mu \leq 1$ formalizes the dependence between the columns of $\boldsymbol{\Phi}$, and can be calculated in polynomial-time complexity.

In order to reconstruct an unknown sparse source from a noisy under-sampled measurement vector, several reconstruction methods have been developed based on convex optimization methods, iterative greedy search algorithms and Bayesian estimation approaches. In this paper, through the design and analysis procedures, we adopt the Bayesian framework [20][24] for reconstructing a sparse source from noisy and quantized measurements.

In the subsequent sections, we describe our proposed design methods for quantization by observing the CS measurement vector, and then develop theoretical results.

\section{JOINT SOURCE-CHANNEL VQ FOR CS}

In this section, we first introduce a general joint sourcechannel VQ system model for CS measurements in Section III-A We derive necessary conditions for optimality of encoder-decoder pair in Section II-B. Thereafter, we investigate the effects of optimal conditions in Section $\amalg$ II-C and proceed to analysis of performance in Section III-D

\section{A. General System Description and Performance Criterion}

Consider the general system model, shown in Figure 1 , for transmitting CS measurements and reconstructing a sparse source. Let the total bit budget allocated for encoding (quantization) be fixed at $R$ bits per dimension of the source vector. Given 
the noisy measurement vector $\mathbf{Y}$, a VQ encoder is defined by a mapping $\mathrm{E}: \mathbb{R}^{M} \rightarrow \mathcal{I}$, where $\mathcal{I}$ is a finite index set defined as $\mathcal{I} \triangleq\left\{0,1, \ldots, 2^{R}-1\right\}$ with $|\mathcal{I}| \triangleq \mathfrak{R}=2^{R}$. Denoting the quantized index by $I$, the encoder works according to $\mathbf{Y} \in \mathcal{R}_{i} \Rightarrow I=i$, where the sets $\left\{\mathcal{R}_{i}\right\}_{i=0}^{\mathfrak{R}-1}$ are encoder regions and $\bigcup_{i=0}^{\mathfrak{R}-1} \mathcal{R}_{i}=\mathbb{R}^{M}$ such that when $\mathbf{Y} \in \mathcal{R}_{i}$ the encoder outputs the index $\mathbf{E}(\mathbf{Y})=i \in \mathcal{I}$. Note that given an index $i$, the set $\mathcal{R}_{i}$ is not necessarily a connected set (due to non-linear CS reconstruction) in the space $\mathbb{R}^{M}$. Also, $\mathcal{R}_{i}$ might be an empty set (due to channel noise, see e.g. [25]).

Next, we consider a memoryless channel consisting of discrete input and output alphabets which is referred to as discrete memoryless channel (DMC). In our problem setup, the DMC accepts the encoded index $i$ and outputs a noisy symbol $j \in \mathcal{I}$. The channel is defined by a random mapping characterized by transition probabilities

$$
P(j \mid i) \triangleq \operatorname{Pr}(J=j \mid I=i), \quad i, j \in \mathcal{I},
$$

which indicates the probability that index $j$ is received given that the input index to the channel was $i$. We assume that the transmitted index $i$ and the received index $j$ share the same index set $\mathcal{I}$, and the channel transition probabilities (3) are known in advance. We denote the capacity of a given channel by $C$ bits/channel use. Given the received index $j$, a decoder is characterized by a mapping $\mathrm{D}: \mathcal{I} \rightarrow \mathcal{C}$ where $\mathcal{C}$ is a finite discrete codebook set containing all reproduction codevectors $\left\{\mathbf{c}_{j} \in \mathbb{R}^{N}\right\}_{j=0}^{\mathfrak{R}-1}$. The decoder's functionality is described by a look-up table; $J=j \Rightarrow \widehat{\mathbf{X}}=\mathbf{c}_{j}$ such that when the received index from the channel is $j$, the decoder outputs $\mathrm{D}(j)=\mathbf{c}_{j} \in \mathcal{C}$.

Next, we state how we quantify the performance of Figure 1 and our design goal. It is important to design an encoderdecoder pair in order to minimize a distortion measure which reflects the requirements of the receiving-end user. Therefore, we quantify the source reconstruction distortion of our studied system by the end-to-end MSE defined as

$$
D \triangleq \mathbb{E}\left[\|\mathbf{X}-\widehat{\mathbf{X}}\|_{2}^{2}\right]
$$

where the expectation is taken with respect to the distributions on the sparse source $\mathbf{X}$ (which, itself, depends on the distribution of non-zero coefficients in $\mathbf{X}$ as well as their random placements (sparsity pattern)), the noise $\mathbf{W}$ and the randomness in the channel. We mention that the end-to-end MSE depends on $C S$ reconstruction error, quantization error as well as channel noise. While the CS sensing matrix $\boldsymbol{\Phi}$ is given, our concern is to design an encoder-decoder pair robust against all these three kinds of error.

\section{B. Optimality Conditions for VQ Encoder and Decoder}

We consider an optimization technique for the system illustrated in Figure 1 in order to determine encoder and decoder mappings $E$ and $D$, respectively, in the presence of channel noise. More precisely, the aim of the VQ design is to find

- MSE-minimizing encoder regions $\left\{\mathcal{R}_{i}\right\}_{i=0}^{\mathfrak{R}-1}$ and

- MSE-minimizing decoder codebook $\mathcal{C}=\left\{\mathbf{c}_{j}\right\}_{j=0}^{\mathfrak{R}-1}$.

We note that the optimal joint design of encoder and decoder cannot be implemented since the resulting optimization is analytically intractable. To address this issue, in Section we show how the encoding index $i \in \mathcal{I}$ (or equivalently encoder region $\mathcal{R}_{i}$ ) can be chosen to minimize the MSE for a given codebook $\mathcal{C}=\left\{\mathbf{c}_{j}\right\}_{j=0}^{\mathfrak{R}-1}$. Then, in Section III-B2 we derive an expression for the optimal decoder codebook $\mathcal{C}$ for given encoder regions $\left\{\mathcal{R}_{i}\right\}_{i=0}^{\mathfrak{R}-1}$.

1) Optimal Encoder: First, let us introduce the minimum mean-square error (MMSE) estimator of the source given the observed measurements (1) which is (see [26, Chapter 11])

$$
\widetilde{\mathbf{x}}(\mathbf{y}) \triangleq \mathbb{E}[\mathbf{X} \mid \mathbf{Y}=\mathbf{y}] \in \mathbb{R}^{N} \text {. }
$$

Now, assume that the decoder codebook $\mathcal{C}=\left\{\mathbf{c}_{j}\right\}_{j=0}^{\mathfrak{R}-1}$ is known and fixed. We focus on how the encoding index $i$ should be chosen to minimize the MSE given the observed noisy CS measurement vector $\mathbf{y}$. We rewrite the MSE as

$$
\begin{aligned}
D & \triangleq \mathbb{E}\left[\|\mathbf{X}-\widehat{\mathbf{X}}\|_{2}^{2}\right]=\mathbb{E}\left[\left\|\mathbf{X}-\mathbf{c}_{J}\right\|_{2}^{2}\right] \\
& \stackrel{(a)}{=} \int_{\mathbf{y}} \sum_{i \in \mathcal{I}} \operatorname{Pr}\{I=i \mid \mathbf{Y}=\mathbf{y}\} \mathbb{E}\left[\left\|\mathbf{X}-\mathbf{c}_{J}\right\|_{2}^{2} \mid \mathbf{Y}=\mathbf{y}, I=i\right] f(\mathbf{y}) d \mathbf{y} \\
& \stackrel{(b)}{=} \sum_{i \in \mathcal{I}} \int_{\mathbf{y} \in \mathcal{R}_{i}}\left\{\mathbb{E}\left[\left\|\mathbf{X}-\mathbf{c}_{J}\right\|_{2}^{2} \mid \mathbf{Y}=\mathbf{y}, I=i\right]\right\} f(\mathbf{y}) d \mathbf{y},
\end{aligned}
$$

where (a) follows from marginalization of the MSE over $\mathbf{Y}$ and $I$. Further, $f(\mathbf{y})$ is the $M$-fold probability density function (pdf) of the measurement vector. Also, (b) follows by interchanging the integral and the summation and the fact that $\operatorname{Pr}\{I=i \mid \mathbf{Y}=\mathbf{y}\}=1, \forall \mathbf{y} \in \mathcal{R}_{i}$, and otherwise the probability is zero. Now, since $f(\mathbf{y})$ is always non-negative, the MSEminimizing points in $\mathbb{R}^{M}$ that shall be assigned to the encoder region $\mathcal{R}_{i}$ are those that minimize the term within the braces in the last expression of (6). Then, the MSE-minimizing encoding index, denoted by $i^{\star} \in \mathcal{I}$, is given by

$$
\begin{aligned}
i^{\star} & =\arg \min _{i \in \mathcal{I}} \mathbb{E}\left[\left\|\mathbf{X}-\mathbf{c}_{J}\right\|_{2}^{2} \mid \mathbf{Y}=\mathbf{y}, I=i\right] \\
& \stackrel{(a)}{=} \arg \min _{i \in \mathcal{I}}\left\{\mathbb{E}\left[\left\|\mathbf{c}_{J}\right\|_{2}^{2} \mid \mathbf{Y}=\mathbf{y}, I=i\right]-2 \mathbb{E}\left[\mathbf{X}^{\top} \mathbf{c}_{J} \mid \mathbf{Y}=\mathbf{y}, I=i\right]\right\} \\
& \stackrel{(b)}{=} \arg \min _{i \in \mathcal{I}}\left\{\mathbb{E}\left[\left\|\mathbf{c}_{J}\right\|_{2}^{2} \mid I=i\right]-2 \mathbb{E}\left[\mathbf{X}^{\top} \mid \mathbf{Y}=\mathbf{y}\right] \mathbb{E}\left[\mathbf{c}_{J} \mid I=i\right]\right\},
\end{aligned}
$$

where $(a)$ follows from the fact that $\mathbf{X}$ is independent of $I$, conditioned on $\mathbf{Y}$; hence, $\mathbb{E}\left[\|\mathbf{X}\|_{2}^{2} \mid \mathbf{Y}=\mathbf{y}, I=i\right]=$ $\mathbb{E}\left[\|\mathbf{X}\|_{2}^{2} \mid \mathbf{Y}=\mathbf{y}\right]$ which is pulled out of the optimization. (b) follows from the fact that $\mathbf{c}_{J}$ is independent of $\mathbf{Y}$, conditioned on $I$, and from the Markov chain $\mathbf{X} \rightarrow \mathbf{Y} \rightarrow I \rightarrow \mathbf{c}_{J}$. Next, note that introducing channel transition probabilities $P(j \mid i)$ in (3) and the MMSE estimator $\widetilde{\mathbf{x}}(\mathbf{y})$ in (5), the last equality in (7) can be expressed as

$$
i^{\star}=\arg \min _{i \in \mathcal{I}}\left\{\sum_{j=0}^{\mathfrak{R}-1} P(j \mid i)\left\|\mathbf{c}_{j}\right\|_{2}^{2}-2 \widetilde{\mathbf{x}}(\mathbf{y})^{\top} \sum_{j=0}^{\mathfrak{R}-1} P(j \mid i) \mathbf{c}_{j}\right\} .
$$

Equivalently, the optimized encoding regions are obtained by

$$
\begin{aligned}
& \mathcal{R}_{i}^{\star}=\{\mathbf{y} \in \mathbb{R}^{M}: \sum_{j=0}^{\Re-1}\left[P(j \mid i)-P\left(j \mid i^{\prime}\right)\right]\left\|\mathbf{c}_{j}\right\|_{2}^{2} \leq \\
&\left.2 \widetilde{\mathbf{x}}(\mathbf{y})^{\top} \sum_{j=0}^{\Re-1}\left[P(j \mid i)-P\left(j \mid i^{\prime}\right)\right] \mathbf{c}_{j}, i \neq i^{\prime} \in \mathcal{I}\right\} .
\end{aligned}
$$


2) Optimal Decoder: Applying the MSE criterion, it is straightforward to show that the codevectors which minimize $D$ in (44) for a fixed encoder are obtained by letting $\mathbf{c}_{j}$ represent the MMSE estimator of the vector $\mathbf{X}$ based on the received index $j$ from the channel, that is

$$
\mathbf{c}_{j}^{\star}=\mathbb{E}[\mathbf{X} \mid J=j], j \in \mathcal{I} .
$$

Now, using the Bayes' rule, the expression for $\mathbf{c}_{j}^{\star}$ can be rewritten as

$$
\begin{aligned}
\mathbf{c}_{j}^{\star} & =\mathbb{E}[\mathbf{X} \mid J=j] \\
& =\sum_{i} P(i \mid j) \mathbb{E}[\mathbf{X} \mid J=j, I=i] \\
& \stackrel{(a)}{=} \frac{\sum_{i} P(j \mid i) P(i) \int_{\mathbf{y}} \mathbb{E}[\mathbf{X} \mid \mathbf{Y}=\mathbf{y}] f(\mathbf{y} \mid i) d \mathbf{y}}{\sum_{i} P(j \mid i) P(i)} \\
& \stackrel{(b)}{=} \frac{\sum_{i} P(j \mid i) \int_{\mathcal{R}_{i}} \widetilde{\mathbf{x}}(\mathbf{y}) f(\mathbf{y}) d \mathbf{y}}{\sum_{i} P(j \mid i) \int_{\mathcal{R}_{i}} f(\mathbf{y}) d \mathbf{y}}
\end{aligned}
$$

where $(a)$ follows from marginalization over $\mathbf{Y}$ and the Markov chain $\mathbf{X} \rightarrow \mathbf{Y} \rightarrow I$. Moreover, $f(\mathbf{y} \mid i)$ is the conditional pdf of $\mathbf{Y}$ given that $\mathbf{Y} \in \mathcal{R}_{i}$. Also, (b) follows by using (5) and by the fact that $f(\mathbf{y} \mid i)=0, \forall \mathbf{y} \notin \mathcal{R}_{i}$.

The optimal conditions in (8) and (11) can be used in an alternate-iterate procedure to design a practical encoderdecoder pair for vector quantization of CS measurements. The resulting algorithm will be presented later in Section IV.

\section{Insights Through Analyzing the Optimal Conditions}

Here, we provide insights into the necessary optimal conditions $(8)$ and (10). Note that the encoding condition (8) implies that the sparse source is first MMSE-wise reconstructed from CS measurements at the encoder, and then quantized to an appropriate index. Hence, it suggests that the system shown in Figure 1 may be translated to the equivalent system shown in Figure 2.

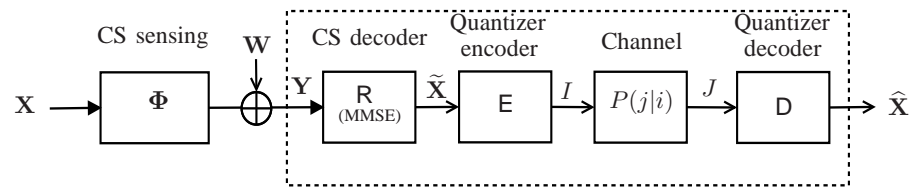

Fig. 2. Equivalent block diagram of a system with CS reconstruction $R$ (MMSE reconstruction) at the encoder side. Necessary optimal conditions for encoder-decoder pair of this system are equivalent to those of the original system model shown in Figure 1.

Let us first denote the MMSE estimator as the RV $\widetilde{\mathbf{X}}(\mathbf{Y}) \triangleq$ $\mathbb{E}[\mathbf{X} \mid \mathbf{Y}]$, then we rewrite the end-to-end distortion $D$ as

$$
\begin{aligned}
D & =\mathbb{E}\left[\|\mathbf{X}-\widetilde{\mathbf{X}}(\mathbf{Y})+\widetilde{\mathbf{X}}(\mathbf{Y})-\widehat{\mathbf{X}}\|_{2}^{2}\right] \\
& =\mathbb{E}\left[\|\mathbf{X}-\widetilde{\mathbf{X}}(\mathbf{Y})\|_{2}^{2}\right]+\mathbb{E}\left[\|\widetilde{\mathbf{X}}(\mathbf{Y})-\widehat{\mathbf{X}}\|_{2}^{2}\right],
\end{aligned}
$$

where the second equality can be proved by showing that the estimation error of the source $\mathbf{X}-\widetilde{\mathbf{X}}(\mathbf{Y})$ and the quantized transmission error $\widetilde{\mathbf{X}}(\mathbf{Y})-\widehat{\mathbf{X}}$ are uncorrelated. This holds from the definition of $\widetilde{\mathbf{X}}(\mathbf{Y})$ and the long Markov property $\mathbf{X} \rightarrow$ $\mathbf{Y} \rightarrow I \rightarrow J \rightarrow \widehat{\mathbf{X}}$ due to the assumption of deterministic mappings $\mathrm{E}$ and $\mathrm{D}$ and memoryless channel.
Remark 1. Following (12), let us denote by $D_{c s} \triangleq \mathbb{E}[\| \mathbf{X}-$ $\left.\widetilde{\mathbf{X}}(\mathbf{Y}) \|_{2}^{2}\right]$ the CS reconstruction distortion, and by $D_{q} \triangleq$ $\mathbb{E}\left[\|\widetilde{\mathbf{X}}(\mathbf{Y})-\widehat{\mathbf{X}}\|_{2}^{2}\right]$ the quantized transmission distortion. Then, the decomposition (12) indicates that the end-to-end source distortion $D$, without loss of optimality, is equivalent to $D=$ $D_{c s}+D_{q}$.

Interestingly, it can be also seen from (12) that $D_{c s}$ does not depend on quantization and channel aspects. Hence, to find optimal encoding indexes (given fixed codevectors) and optimal codevectors (given fixed encoding regions) with respect to the end-to-end distortion $D$, it suffices to find them with respect to minimizing $D_{q}$. It can be proved that the necessary conditions for optimality (with respect to $D_{q}$ ) of the encoder-decoder pair derived for the system of Figure 2 coincide with the ones developed for the system of Figure 11 i.e., (8) and (11). The proof of this claim is as follows. Similar to the steps taken in (6), the $D_{q}$-minimizing encoding index $i^{\star} \in \mathcal{I}$ is given by

$$
\begin{aligned}
i^{\star} & =\arg \min _{i \in \mathcal{I}} \mathbb{E}\left[\left\|\widetilde{\mathbf{X}}(\mathbf{Y})-\mathbf{c}_{J}\right\|_{2}^{2} \mid \mathbf{Y}=\mathbf{y}, I=i\right] \\
& =\arg \min _{i \in \mathcal{I}}\left\{\mathbb{E}\left[\left\|\mathbf{c}_{J}\right\|_{2}^{2} \mid I=i\right]-2 \widetilde{\mathbf{x}}(\mathbf{y})^{\top} \mathbb{E}\left[\mathbf{c}_{J} \mid I=i\right]\right\}, \\
& =\arg \min _{i \in \mathcal{I}}\left\{\sum_{j=0}^{\Re-1} P(j \mid i)\left\|\mathbf{c}_{j}\right\|_{2}^{2}-2 \widetilde{\mathbf{x}}(\mathbf{y})^{\top} \sum_{j=0}^{\Re-1} P(j \mid i) \mathbf{c}_{j}\right\} .
\end{aligned}
$$

Further, the $D_{q}$-minimizing decoder $\mathbf{c}_{j}^{\star}$ is obtained by

$$
\begin{aligned}
\mathbf{c}_{j}^{\star} & =\mathbb{E}\left[\widetilde{\mathbf{X}}^{\star}(\mathbf{Y}) \mid J=j\right] \\
& \stackrel{(a)}{=} \int \mathbb{E}[\mathbf{X} \mid J=j, \mathbf{Y}=\mathbf{y}] p(\mathbf{y} \mid j) d \mathbf{y} \\
& =\mathbb{E}[\mathbf{X} \mid J=j],
\end{aligned}
$$

where $(a)$ follows from the Markov property $\widetilde{\mathbf{X}}(\mathbf{Y}) \rightarrow \mathbf{Y} \rightarrow J$. Now, we provide the following remark.

Remark 2. The general system of Figure $\square$ and Figure 2 are equivalent considering end-to-end MSE criterion, fixed sensing matrix and channel transition probabilities.

Before proceeding to the analysis of the MSE using the developed equivalence property, we provide a comparative study between our proposed design scheme with related methods in the literature which follow the building block structure shown in Figure 3 Under this system model, for a fixed CS reconstruction algorithm (or, a fixed quantizer encoder-decoder pair), a quantizer encoder-decoder pair (or, CS reconstruction algorithm) is designed in order to satisfy a certain performance criterion, e.g. minimizing end-to-end distortion, quantization distortion or $\ell_{1}$-norm of reconstruction vector. Some examples of system models following Figure 3 include [3], [5], [9], [11] (assuming a noiseless channel) and the conventional nearestneighbor coding of CS measurements. In general, according to this system model, quantizer decoder $\mathrm{D}$ outputs the vector $\widehat{\mathbf{Y}} \in \mathbb{R}^{M}$ after receiving channel output. Finally, a given CS reconstruction decoder $\mathrm{R}: \mathbb{R}^{M} \rightarrow \mathbb{R}^{N}$ takes $\widehat{\mathrm{Y}}$ and makes an estimate of the sparse source.

Following Figure 2 (as the equivalent system model of Figure 1), we note that it is structurally different from the system model of Figure 3 in the location of the CS reconstruction, 


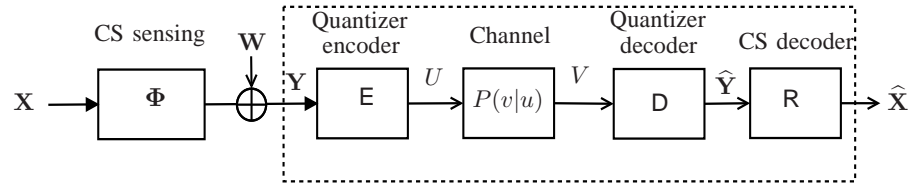

Fig. 3. Block diagram of a system with CS reconstruction at the decoder side. The aim is to design encoder-decoder mappings or CS decoder (illustrated in dashed box) with respect to optimizing a performance criterion while the CS sensing matrix $\boldsymbol{\Phi}$ and channel $P(v \mid u)$ are known in advance.

either at the transmitter side or at the receiver side. In the former system, an encoder reconstructs the source from CS measurements, whereas the latter system puts all CS reconstruction complexity at the decoder.

\section{Analysis of MSE}

In this section, we provide an analysis into the impact of CS reconstruction distortion, quantization error and channel noise on the end-to-end MSE by deriving a lower-bound.

Proposition 1. Consider the linear CS model 11 with an exact $K$-sparse source $\mathbf{X} \in \mathbb{R}^{N}$ under the following assumptions:

$i$. The magnitude of $K$ non-zero coefficients in $\mathbf{X}$ are drawn according to the i.i.d. standard Gaussian distribution.

ii. The $K$ elements of the support set are uniformly drawn from all $\left(\begin{array}{l}N \\ K\end{array}\right)$ possibilities.

iii. The measurement noise is drawn as $\mathbf{W} \sim \mathcal{N}\left(\mathbf{0}, \sigma_{w}^{2} \mathbf{I}_{M}\right)$ uncorrelated with the measurements, where $\sigma_{w}^{2} \neq 0$.

Further, assume a sensing matrix $\mathbf{\Phi}$ with mutual coherence $\mu$. Let the total quantization rate be $R$ bits/vector, and the channel be characterized by capacity $C$ bits/channel use, then the end-to-end MSE of the system of Figure 1 asymptotically (in quantization rate and dimension) is lower-bounded as

$$
D \geq K c_{1}+c_{1} c_{2} 2^{-2 C\left(\frac{R-\log _{2}\left(\begin{array}{l}
N \\
K
\end{array}\right)}{K}\right)},
$$

where $c_{1}=\frac{\sigma_{w}^{2}}{1+\sigma_{w}^{2}+(K+1) \mu}$, and $c_{2}=2\left(\frac{K}{2} \Gamma\left(\frac{K}{2}\right)\right)^{\frac{2}{K}}\left(\frac{K+2}{K}\right)^{\frac{K}{2}}$, in which $\Gamma(\cdot)$ denotes the Gamma function.

Proof: The proof can be found in the Appendix.

Remark 3. Each component of the lower-bound (13) is intuitive. The first term is the contribution of the CS reconstruction distortion, and the second term reflects the distortion due to the vector quantized transmission. When the CS measurements are noisy, it can be verified that as $R$ increases, the end-to-end MSE attains an error floor. This result can be also inferred from (12): as quantization rate increases, $D_{q}$ decays (asymptotically) exponentially, however, $D_{c s}$ is constant irrespective of rate. Hence, as $R \rightarrow \infty$, the value that the MSE converges to is $D_{c s}=\mathbb{E}\left[\|\mathbf{X}-\widetilde{\mathbf{X}}\|_{2}^{2}\right]$.

It should be noted when CS measurements are noiseless $\left(\sigma_{w}^{2}=0\right)$, the lower-bound (13) becomes trivial. In this case, a simple asymptotic lower-bound for the system of Figure 1 under the assumptions of Proposition 1 can be obtained as

$$
D \geq c_{2} 2^{-2 C\left(\frac{R-\log _{2}\left(\begin{array}{l}
N \\
K
\end{array}\right)}{K}\right)},
$$

where the constant $c_{2}$ is the same dimensionality-dependent constant in (13).

The lower-bound (14) (also known as adaptive bound in [16], [17] in the noiseless channel case) can be proved assuming that the support set of $\mathbf{X} \in \mathbb{R}^{N}$ is a priori known. Therefore, one can transmit the known support set using $\log _{2}\left(\begin{array}{l}N \\ K\end{array}\right)$ bits, and the Gaussian coefficients within the support set can be quantized via $R-\log _{2}\left(\begin{array}{l}N \\ K\end{array}\right)$ bits. Under noiseless channel condition $(C=1)$, the right hand side in (14) is shown to achieve the distortion rate function of a $K$-sparse source vector with Gaussian nonzero coefficients and a support set uniformly drawn from $\left(\begin{array}{l}N \\ K\end{array}\right)$ possibilities [27]. Then, the separate source-channel coding theorem [28, Chapter 7] can be applied to find the optimum performance theoretically attainable (OPTA) by introducing channel capacity $C$.

Remark 4. The lower-bound in (14) shows that the end-to-end MSE can at most decay exponentially (in quantization rate $R$ ) with exponent $-\frac{6 C}{K} \mathrm{~dB} /$ bit. Since the sparsity ratio $\frac{K}{N}<1$, the decaying exponent can be far steeper than $-\frac{6 C}{N} \mathrm{~dB} / \mathrm{bit}$ for a Gaussian non-sparse source vector of dimension $N$.

The following toy example offers some insights into the tightness of the lower-bound (14).

Example 1. Using a simple example, we show how tight the lower-bound (14) is with respect to our proposed design. In Figure 4 we compare simulation results with the lower-bound in some region where $\widetilde{\mathbf{X}}(\mathbf{Y}) \rightarrow \mathbf{X} \cdot 1$ Following this best-case scenario, we generate $2 \times 10^{5}$ realizations of $\mathbf{X} \in \mathbb{R}^{2}$ with sparsity level $K=1$, where the non-zero coefficient is a standard Gaussian $R V$, and its location is drawn uniformly at random over $\{1,2\}$. Then, we use the necessary optimal conditions (8) and (10) iteratively (as will be shown later in Algorithm 1]. Considering a binary symmetric channel (BSC) with bit cross-over probability $\epsilon$ and capacity $C$ bits/channel use (see (29)), we plot MSE, $D=\mathbb{E}\left[\|\mathbf{X}-\widehat{\mathbf{X}}\|_{2}^{2}\right]$ versus quantization rate $R$ for $\epsilon=0$ (noiseless channel) and $\epsilon=0.02$ (noisy channel) in Figure 4 It can be observed that at $\epsilon=0$, the bound (dashed line) is tight. As would be expected, degrading channel condition to $\epsilon=0.02$ reduces the performance. At $\epsilon=0.02$, the gap between the simulation result (solid line marked by 'o') and its corresponding lower-bound (dotted line) increases. Note that in the noisy channel case, the lower-bound is based upon the asymptotic assumption of infinite source and channel code lengths (used in the OPTA). Therefore, the lower-bound is not tight at $\epsilon=0.02$ for low dimensions.

\section{Practical Quantizer Design}

In this section, we first develop a practical VQ encoderdecoder design algorithm, referred to as channel-optimized VQ for CS (COVQ-CS) using the necessary optimal conditions (8) and 111). Then, we provide a practical comparison between our proposed algorithm and a conventional quantizer design algorithm. We finalize this section by analyzing encoding and decoding computational complexity.

\footnotetext{
${ }^{1}$ This scenario can be realized in an event where $\sigma_{w}^{2}=0$ and number of measurements is such that the CS reconstruction is perfect.
} 


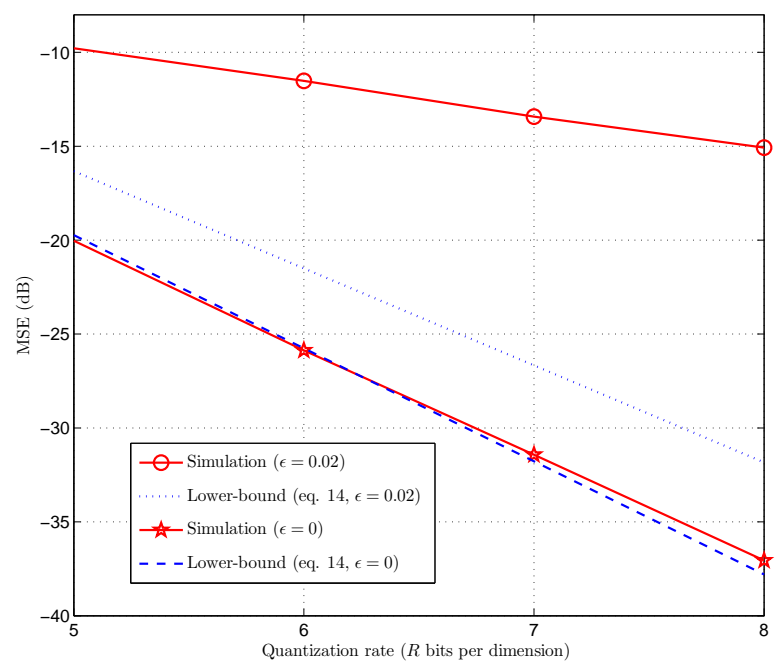

Fig. 4. Comparison of the lower-bound in 14 and simulation results for simulation of a 1-sparse source $\mathbf{X} \in \mathbb{R}^{2}$ in a region where the locally reconstructed source $\widetilde{\mathbf{X}}$ can be perfectly recovered from noiseless measurements.

\section{A. Training Algorithm for Practical Design}

The results presented in Section $\amalg I-B 1$ and Section III-B2 can be utilized to formulate an iterate-alternate training algorithm for the problem of interest. Similar to the generalized Lloyd algorithm for noisy channels [29], we propose a VQ training method for the design problem in this paper which is summarized in Algorithm 1 The following remarks can be considered for implementing Algorithm 1

- In step (1), besides the channel transition probabilities $P(j \mid i)$, we assume that the statistics of the sparse source vector are given for training.

- In general, it is not easy to derive closed-form solutions for the optimal decoding condition (11), for example, due to difficulties in calculating the integrals even if the pdf $f(\mathbf{y})$ is known. In practice, we calculate the codevector $\mathbf{c}_{j}(j \in$ $\mathcal{I})$ in (10) using the Monte-Carlo method. To implement this computationally-efficient procedure, we first generate a set of finite training vectors $\mathbf{X}$, and then sample-average over those vectors that have led to the index $J=j$.

- To address the issue of encountering empty regions, we, in each iteration of the algorithm, pick the codevector whose index has been sent the most number of times, denoted by $\mathbf{c}_{j}^{\max }$. Then, a codevector associated with the index that has not been sent is calculated as $\mathbf{c}_{j}^{\max }+\delta \mathbf{c}_{j}^{\max }$, where $\delta>0$ is sufficiently small. Using this technique (which is also known as splitting method in the initialization phase of the LBG algorithm [30]), we efficiently re-include those encoding indexes that have never been selected due to the limited number of generated samples. This will lead to a design that efficiently uses all degrees of freedom.

- The performance of the COVQ-CS is sensitive to initializations in order for the algorithm to converge to a smaller value of the distortion $D$. Therefore, in step (3), when the channel is noiseless, the codevectors are initialized using the splitting procedure of the so-called LBG design algorithm. Then, the final optimized codevectors are chosen for initialization of Algorithm 1 in the noisy channel case. Furthermore, convergence in step (7) may be checked by tracking the MSE, and terminate the iterations when the relative improvement is small enough. By construction and ignoring issues such as numerical precision, the iterative design in Algorithm 1 always converges to a local optimum since when the criteria in steps (5) and (6) of the algorithm are invoked, the performance can only leave unchanged or improved, given the updated indexes and codevectors. This is a common rationale behind the proof of convergence for such iterative algorithms (see e.g. [31, Lemma 11.3.1]). However, nothing can be generally guaranteed about the global optimality of this algorithm.

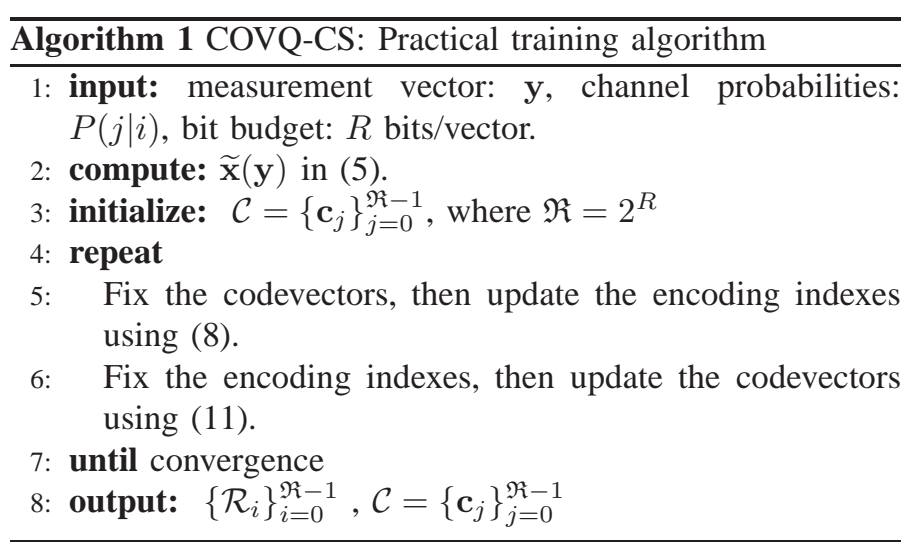

\section{B. Practical Comparison}

Here, we offer further insights into quantization aspects through the design of conventional nearest-neighbor coding (NNC) as a representative of Figure 3, and the design of proposed COVQ-CS method as a representative of Figure 2. The NNC for CS is often considered as a benchmark for performance evaluations.

The nearest-neighbor coding (NNC) for CS measurements is accomplished by designing a channel-optimized VQ for the input vector $\mathbf{Y}$ aiming to minimize the quantization distortion, i.e., $\mathbb{E}\left[\|\mathbf{Y}-\widehat{\mathbf{Y}}\|_{2}^{2}\right]$, where $\widehat{\mathbf{Y}} \in \mathbb{R}^{M}$ is the quantizer decoder output as shown in Figure 3$]$ Considering the notations given for the Figure 3 , the design procedure of the quantizer encoder and the quantizer decoder is as follows: for a quantization rate $R$ bits/vector, a fixed codebook $\mathcal{G}=\left\{\mathbf{g}_{v} \in \mathbb{R}^{M}\right\}_{v=0}^{\mathfrak{R}-1}$, with $\mathfrak{R}=$ $2^{R}$, and channel transition probability $P(v \mid u)$, the optimized encoding region $\mathcal{R}_{u}^{\star}$ becomes

$$
\begin{aligned}
\mathcal{R}_{u}^{\star}= & \left\{\mathbf{y} \in \mathbb{R}^{M}: \sum_{v=0}^{\Re-1}\left[P(v \mid u)-P\left(v \mid u^{\prime}\right)\right]\left\|\mathbf{g}_{v}\right\|_{2}^{2} \leq\right. \\
& \left.2 \mathbf{y}^{\top} \sum_{v=0}^{\Re-1}\left[P(v \mid u)-P\left(v \mid u^{\prime}\right)\right] \mathbf{g}_{v}, u \neq u^{\prime} \in \mathcal{U}\right\},
\end{aligned}
$$

${ }^{1}$ See e.g. [29] for more details regarding the design of channel-optimized VQ in a non-CS system model. 


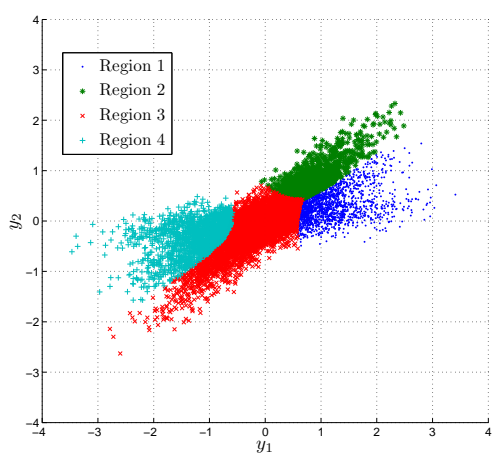

(a) Encoding regions using the proposed COVQ-CS 9] in $\mathbb{R}^{2}$

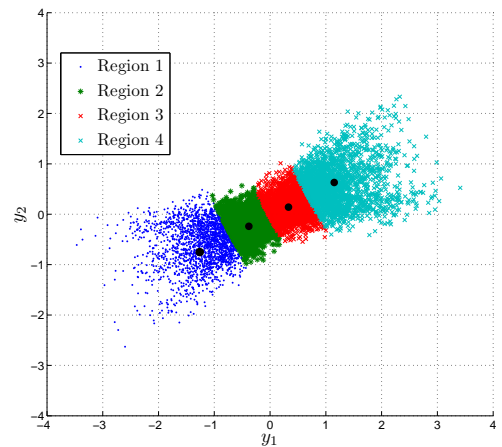

(c) Encoding regions using the NNC-CS 15 in $\mathbb{R}^{2}$

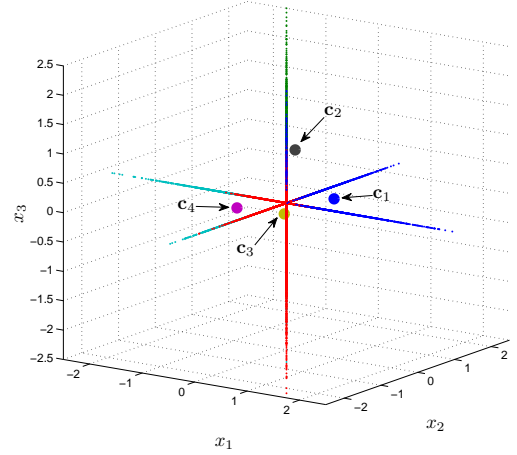

(b) Codevectors designed by COVQ-CS in $\mathbb{R}^{3}$

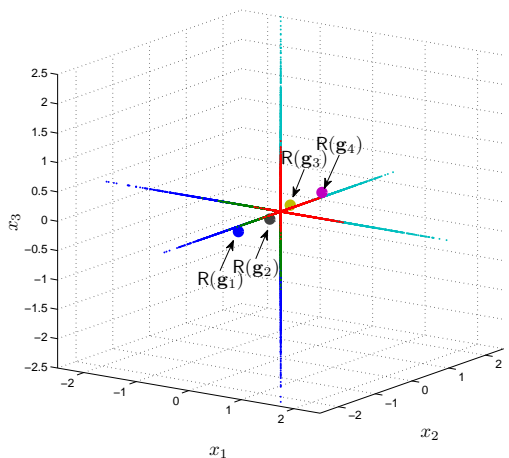

(d) Reconstructed Codevectors designed by NNC-CS in $\mathbb{R}^{3}$

Fig. 5. The qualitative behavior comparison of quantizer schemes: COVQ-CS and NNC-CS designed for a BSC with $\epsilon=0.02$.

where $\mathcal{U} \triangleq\left\{0, \ldots, 2^{R}-1\right\}$ is the encoding index set. Now, for the given region (15) and channel transition probability $P(j \mid i)$, the quantization MSE-minimizing codevectors satisfy

$$
\mathbf{g}_{v}^{\star}=\mathbb{E}[\mathbf{Y} \mid V=v], \quad v \in \mathcal{U} .
$$

In order to design an encode-decoder pair using the $\mathrm{NNC}$, an iterative algorithm can be used to alternate between (15) and (16). Finally, a CS reconstruction algorithm $R$ produces the reconstruction vector $\widehat{\mathbf{X}}$ from the quantizer decoder output $\widehat{\mathbf{Y}}$. We refer to this design method as NNC-CS.

Example 2. In this example, we illustrate how the COVQCS and NNC-CS design methods are different in shaping encoding regions (given that CS measurements are observed) and positioning codevectors (given that channel output index observed). For illustration purpose, we choose the input sparse vector dimension, measurement vector dimension and sparsity level as $N=3, M=2$ and $K=1$, respectively. The location of non-zero coefficient is drawn uniformly at random from $\{1, \ldots, N\}$, and its value is a standard Gaussian $R V$. For implementing the COVQ-CS via Algorithm $\square$ the MMSE estimator $\widetilde{\mathbf{x}}(\mathbf{y})$ (used in (8)) is calculated via the closed-form solution given in [22. eq. (27)]. We generate $10^{4}$ realizations for $\mathbf{X}$ (and subsequently $\mathbf{Y}$ ), where measurement noise vector is drawn from $\mathcal{N}\left(\mathbf{0}, \sigma_{w}^{2} \mathbf{I}_{M}\right)$ with $\sigma_{w}^{2}=0.04$. Then, we fix the quantization rate at $R=2$ bits/vector and assume a BSC with cross-over probability $\epsilon=0.02$. For implementing the NNC-CS, an iterative algorithm is used by alternating between encoding regions (15) and codevectors (16). Finally, a CS reconstruction algorithm $R: \mathbb{R}^{M} \rightarrow \mathbb{R}^{N}$ (here, we choose the same MMSE estimator used at the encoder of COVQ-CS) takes the NNC-CS codevectors and produces an estimate of the sparse source. In both NNC-CS and COVQ-CS schemes, the sensing matrix $\mathbf{\Phi}$ is chosen as

$$
\mathbf{\Phi}=\left(\begin{array}{lll}
0.9924 & 0.8961 & 0.7201 \\
0.1230 & 0.4439 & 0.6939
\end{array}\right) \text {. }
$$

In Figure 5 we qualitatively illustrate encoding regions and codevectors using the two designs. Figure 5(a) shows the samples of CS measurements classified by encoding regions of COVQ-CS in $\mathbb{R}^{2}$, i.e., (8), and Figure 5(b) shows the samples of $\mathrm{X}$ classified by the index of encoding regions (in the same color) together with the codevectors of COVQ-CS in $\mathbb{R}^{3}$, i.e., $\left\{\mathbf{c}_{j}\right\}_{j=1}^{4}$ in $11 \mathrm{~b}$. Figure $5(\mathrm{c})$ illustrates the encoding regions of NNC-CS, i.e., [15), together with codevectors $\left\{\mathbf{g}_{v}\right\}_{v=1}^{4}$ shown by black circles, and Figure $5(d)$ shows the samples of the sparse source along with the codevectors of NNC-CS mapped to the 3-dimensional space using the CS reconstruction algorithm, i.e., $R\left(\left\{\mathbf{g}_{v}\right\}_{v=1}^{4}\right)$. From the samples in the measurement space, we observe that the entries of the CS measurements are highly correlated, in this particular example, due to a large mutual coherence of the sensing matrix ( $\mu=0.9533)$. Hence, as shown in Figure 5(c), the codevectors designed by the NNC-CS (almost) lie on a single line. Although, in this case, the location of code- 
vectors are optimized to minimize the quantization distortion, $\mathbb{E}\left[\|\mathbf{Y}-\widehat{\mathbf{Y}}\|_{2}^{2}\right]$, it is critical when the codevectors are mapped back to the source domain. From Figure $5(d)$ it is observed that the reconstructed codevectors, $R\left(\left\{\mathbf{g}_{v}\right\}_{v=1}^{4}\right)$, are not only situated (approximately) on one axis but also far (in Euclidean distance) to their corresponding source samples (shown in same color) resulting in a high end-to-end distortion. Further, if, for example, the codevector $\mathbf{g}_{1}$ is received as $\mathbf{g}_{4}$ due to channel noise, it produces a large end-to-end distortion. Using other experiments, in the case of noiseless channel, we observed the same trend in the location of reconstructed codevectors (using $N N C-C S)$ on the source domain which also produces large MSE in terms of the average distance between source samples and their corresponding reconstructed codevectors. While this is the case in NNC-CS, it can be seen from Figure 5(a) that the encoding regions using COVQ-CS may not form convex sets (for example, region 3) unlike the ones using the NNC-CS. This is due to the fact that the region fixed by the rule (9) may not be a convex set in $\mathbf{y}$ due to non-linearity in $\widetilde{\mathbf{x}}(\mathbf{y})$. As a result, the COVQ-CS uses the measurement space more efficiently in order to reduce end-to-end distortion, $\mathbb{E}\left[\|\mathbf{X}-\widehat{\mathbf{X}}\|_{2}^{2}\right]$. It can be observed from Figure $5(b)$ that the COVQ-CS codevectors are located on different coordinates in the 3-dimensional source space to minimize the end-to-end source distortion. In addition, the codevectors are located such that the COVQ-CS design becomes more robust against channel noise which produces smaller end-to-end distortion unlike the NNC-CS design. For example, as shown in Figure 5(b), if the codevector $\mathbf{c}_{1}$ is chosen as $\mathbf{c}_{4}$ at decoder due to channel noise, it provides much less end-to-end distortion than that of the NNC-CS. Numerical performance comparison between these two schemes will be made later in Section VI-B through different simulation studies.

\section{Complexity of COVQ-CS}

We analyze the encoding computational complexity (time usage) as well as encoder-decoder memory complexity (space usage) for the COVQ-CS. For encoding computational complexity, we calculate the number of operations (in terms of FLOH) required for transmitting an encoded index over the channel based on (8). In addition, for memory complexity, we calculate the memory (in terms of floa 2 ) required for storing vector parameters at encoder and decoder.

The encoding complexity for computing the argument in (8) requires one FLOP for calculating the subtraction as well as $2 N-1$ FLOP's ( $N$ multiplications and $N-1$ additions) for calculating the inner product in the second term. Thus, the total complexity for the full-search minimization at encoder is $22^{R}$ FLOP's. Note that we do not consider the complexity of CS reconstruction algorithm since its calculation is required for all relevant quantizers for CS. Next, considering the argument in (8), the encoder needs one float to store the first constant term in (8), i.e., $\left\|\mathbf{c}_{j}\right\|_{2}^{2}$, and also $N$ floats to store the second term in (8), i.e., the codevector $\mathbf{c}_{j}$. Thus, the total encoding memory

\footnotetext{
${ }^{1}$ Each addition, multiplication and comparison is represented by one floating point operation (FLOP).

${ }^{2}$ Float is considered as a single precision point unit.
}

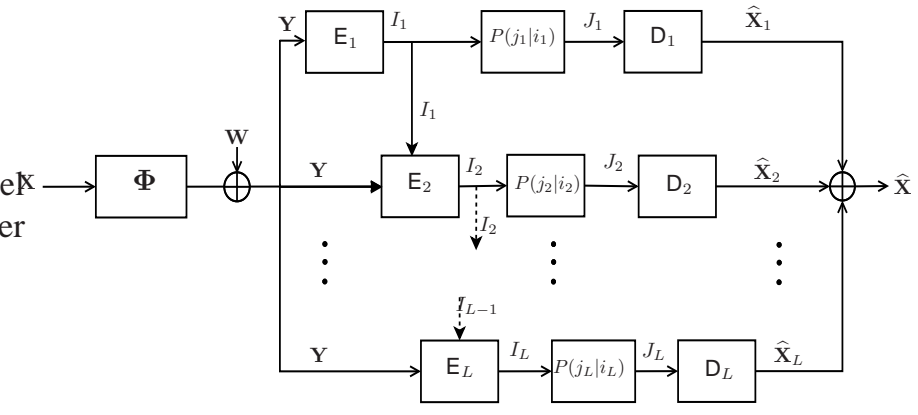

Fig. 6. JSCC system model for CS measurements using MSVQ.

for full-search minimization is $(N+1) 2^{R}$. It also follows that the decoder requires $N 2^{R}$ floats to store $\mathbf{c}_{j}$ in (10).

Using high-dimensional VQ and CS, the implementation of the quantizer encoder and decoder may not be feasible, both from computational complexity and from memory complexity viewpoints. The complexity can be reduced by exploiting suboptimal approaches (with respect to (4)) such as multi-stage VQ (MSVQ) which splits a single VQ into multiple VQ's at different stages. In the next section, we focus on the design of JSCC strategies for CS measurements using MSVQ.

\section{JoInt SOURCE-ChANNEL MSVQ FOR CS}

Taking advantage of VQ properties by addressing its encoding complexity effectively has led to development of multi-stage VQ (MSVQ).

\section{A. System Description and Performance Criterion}

In this section, we give an account for the basic assumptions and models made about the investigated system depicted in Figure 6. We illustrate an $L$-stage VQ, where $L \geq 1$ is the maximum number of stages. Our MSVQ system model basically follows that of [32]. More specifically, we consider the $l^{\text {th }}(1 \leq l \leq L)$ stage with allocated $R_{l}$ bits/vector, where $\sum_{l=1}^{L} R_{l}=R$, and $R$ is the total available quantization rate. Indeed, $R_{l}$ adjusts a trade-off between complexity and performance of MSVQ. A quantizer encoder, at stage $l$, accepts the measurement vector $\mathbf{Y}$ and the encoded index from the $(l-1)^{t h}$ stage as inputs, then maps them into an integer index $i_{l} \in \mathcal{I}_{l} \triangleq\left\{0, \ldots, 2^{R_{l}}-1\right\}$ with $\left|\mathcal{I}_{l}\right| \triangleq \mathfrak{R}_{l}=2^{R_{l}}$. Therefore, the $l^{\text {th }}$-stage encoder is described by a mapping $\mathrm{E}_{l}: \mathbb{R}^{M} \times \mathcal{I}_{l-1} \rightarrow \mathcal{I}_{l}$ such that

$$
\mathrm{E}_{l}\left(\mathbf{Y}, I_{l-1}\right)=i_{l}, \quad \text { if }\left(\mathbf{Y} \in \mathcal{R}_{i_{1}}^{i_{l}}, I_{l-1}=i_{l-1}\right),
$$

where $\mathcal{R}_{i_{1}}^{i_{l}} \triangleq \mathcal{R}_{i_{1}} \cap \ldots \cap \mathcal{R}_{i_{l}}$ is called the $l^{t h}$-stage encoding region. The region $\mathcal{R}_{i_{1}}^{i_{l}}$ might be a connected set or union of some connected sets in $\mathbb{R}^{M}$. We also make the assumption that $\mathcal{I}_{0}=\varnothing$.

The encoded index $I_{l}$ is transmitted over a DMC (independent of other channels) with transition probabilities

$$
P\left(j_{l} \mid i_{l}\right)=\operatorname{Pr}\left(J_{l}=j_{l} \mid I_{l}=i_{l}\right), \quad i_{l}, j_{l} \in \mathcal{I}_{l},
$$

where $J_{l}$ denotes the channel output at the $l^{\text {th }}$ stage.

Next, a decoder $\mathrm{D}_{l}$ accepts the noisy index $J_{l}$, and provides an estimation of the quantization error according to an available 
codebook set. Formally, the $l^{\text {th }}$-stage decoder is defined by a mapping $\mathrm{D}_{l}: \mathcal{I}_{l} \rightarrow \mathcal{C}_{l}$ where $\mathcal{C}_{l}$ denotes a codebook set consists of reproduction codevectors, i.e., $\mathcal{C}_{l} \triangleq\left\{\mathbf{c}_{j_{l}} \in \mathbb{R}^{N}\right\}_{j_{l}=0}^{\mathfrak{R}_{l}-1}$, thus

$$
\mathrm{D}_{l}\left(j_{l}\right)=\mathbf{c}_{j_{l}}, \quad \text { if } J_{l}=j_{l}, \quad j_{l} \in \mathcal{I}_{l} .
$$

We denote the output of the $l^{t h}$ stage decoder by $\widehat{\mathbf{X}}_{l}=\mathbf{c}_{J_{l}}$, and the final reconstructed vector by $\widehat{\mathbf{X}}=\sum_{l=1}^{L} \widehat{\mathbf{X}}_{l}$.

We are interested in designing the quantizers in the system of Figure 6 using the end-to-end MSE criterion defined in (4). Nevertheless, it is not easy to find optimal encoders (by fixing the decoders) and decoders (by fixing encoders) for all the stages jointly with respect to minimizing (4). Therefore, we define a new performance criterion as

$$
D_{l} \triangleq \mathbb{E}\left[\left\|\mathbf{X}-\sum_{t=1}^{l} \widehat{\mathbf{X}}_{t}\right\|_{2}^{2}\right], \quad l=1, \ldots, L
$$

Using the performance criterion $D_{l}$ in (20), we assume that the $l^{\text {th }}$ stage only observes the previous $(l-1)$ stages. Applying $D_{l}$, we derive necessary encoding and decoding policies for optimality (with respect to (20) at stage $l(1 \leq l \leq L)$. Then, encoder-decoder pairs at the next stages are sequentially designed one after another. Using the sequential optimization at stage $l$, we assume that the subsequent codevectors are populated with zero. This assumption means that the sequential design is sub-optimal with respect to (4), and the resulting conditions would lead to neither global nor local minimum of the end-to-end MSE. However, it provides better performance compared to the schemes which only consider quantization distortion at each stage separately.

\section{B. Optimality Conditions for MSVQ Encoder and Decoder}

In this section, we develop encoding and decoding principles for the $l^{t h}(1 \leq l \leq L)$ stage of the MSVQ system shown Figure 6 Following the arguments of Section III-B we first assume that decoder codevectors $\left\{\mathbf{c}_{j_{l}}\right\}_{j_{l}=0}^{\mathfrak{R}_{l}-1}$ and all encoding regions/codevectors at previous $l-1$ stages are fixed and known, then we find necessary optimal encoding regions with respect to minimizing $D_{l}$ in 20 in Section V-B1 Second, we fix the encoding regions $\left\{\mathcal{R}_{i_{1}}^{i_{l}}\right\}$, and then derive necessary optimal codevectors in Section V-B2. Finally, in Section V-B3. we combine these necessary optimal conditions to develop a practical MSVQ design algorithm referred to as channeloptimized MSVQ for CS (COMSVQ-CS).

1) Optimal Encoder: In order to derive encoding regions $\left\{\mathcal{R}_{i_{1}}^{i_{l}}\right\}$, we fix the codevectors $\left\{\mathbf{c}_{j_{l}}\right\}_{j_{l}=0}^{\mathfrak{R}_{l}-1}$ and all the codevectors at previous stages. First, let us define

$$
D_{l}\left(\mathbf{y}, \mathbf{i}_{1}^{l}\right) \triangleq \mathbb{E}\left[\left\|\mathbf{X}-\sum_{t=1}^{l} \widehat{\mathbf{X}}_{t}\right\|_{2}^{2} \mid \mathbf{Y}=\mathbf{y}, \mathbf{I}_{1}^{l}=\mathbf{i}_{1}^{l}\right], \quad 1 \leq l \leq L .
$$

Now, $D_{l}$ in 20 can be rewritten as

$$
\begin{aligned}
D_{l} & \triangleq \mathbb{E}\left[\left\|\mathbf{X}-\sum_{t=1}^{l} \mathbf{c}_{J_{t}}\right\|_{2}^{2}\right] \\
& \stackrel{(a)}{=} \sum_{i_{1}, \ldots, i_{l}} \int_{\mathcal{R}_{i_{1}}^{i_{l}}} D_{l}\left(\mathbf{y}, \mathbf{i}_{1}^{l}\right) f(\mathbf{y}) d \mathbf{y},
\end{aligned}
$$

where $(a)$ follows from marginalization of $D_{l}$ over $\mathbf{Y}$ and $I$, and the fact that $\operatorname{Pr}\left\{\mathbf{I}_{1}^{l}=\mathbf{i}_{1}^{l} \mid \mathbf{Y}=\mathbf{y}\right\}=1, \forall \mathbf{y} \in \mathcal{R}_{i_{1}}^{i_{l}}$ and otherwise the probability is zero.

Thus, $\forall i_{1}, \ldots, i_{l-1}$, the optimized index, denoted by $i_{l}^{\star}$, is attained by (23), where $(a)$ follows from the Markov chain $\mathbf{X} \rightarrow\left(\mathbf{Y}, \mathbf{I}_{1}^{t-1}\right) \rightarrow I_{t}(\forall t \in\{1, \ldots, l\})$, hence, $\mathbb{E}\left[\|\mathbf{X}\|_{2}^{2} \mid \mathbf{Y}=\mathbf{y}, \mathbf{I}_{1}^{l}=\mathbf{i}_{1}^{l}\right]=\mathbb{E}\left[\|\mathbf{X}\|_{2}^{2} \mid \mathbf{Y}=\mathbf{y}\right]$ which is pulled out of the optimization. Also, $(b)$ follows from the Markov chain $\left(\mathbf{Y}, \mathbf{I}_{1}^{l}\right) \rightarrow I_{t} \rightarrow \mathbf{c}_{J_{t}}, \forall t \in\{1, \ldots, l\}$.

Introducing transition probabilities (18) and the MMSE estimator (5), the last equality in 23) is expressed as

$$
\begin{aligned}
i_{l}^{\star} & =\arg \min _{i_{l} \in \mathcal{I}_{l}}\left\{\sum_{j_{l}=0}^{\Re_{l}-1} P\left(j_{l} \mid i_{l}\right)\left\|\mathbf{c}_{j_{l}}\right\|_{2}^{2}-2 \widetilde{\mathbf{x}}(\mathbf{y})^{\top} \sum_{j_{l}=0}^{\mathfrak{R}_{l}-1} P\left(j_{l} \mid i_{l}\right) \mathbf{c}_{j_{l}}\right. \\
& \left.+2 \sum_{j_{l}=0}^{\mathfrak{R}_{l}-1} \sum_{t=1}^{l-1} \sum_{j_{t}=0}^{\Re_{t}-1} P\left(j_{l} \mid i_{l}\right) P\left(j_{t} \mid i_{t}\right) \mathbf{c}_{j_{l}}^{\top} \mathbf{c}_{j_{t}}\right\} .
\end{aligned}
$$

Remark 5. Comparing the optimized encoding index for MSVQ for CS in (24), with that of the VQ for CS in (8), it can be seen that the third term in 24 is due to imposing multi-stage structure on the original VQ. As $L=1$, this term vanishes and the resulting expression coincides with (8).

2) Optimal Decoder: In order to derive codevectors $\left\{\mathbf{c}_{j_{l}}\right\}_{j_{l}=0}^{\mathfrak{R}_{l}-1}$, we fix encoding regions $\left\{\mathcal{R}_{i_{1}}^{i_{l}}\right\}$ and all prior codebook sets. Therefore, applying $D_{l}$ in (20), it is straightforward to show that the optimal $l$-stage codevectors, denoted by $\left\{\mathbf{c}_{j_{l}}^{\star}\right\}_{j_{l}=1}^{\mathfrak{R}_{l}-1}$, are obtained as

$$
\mathbf{c}_{j_{l}}^{\star}=\mathbb{E}\left[\mathbf{X}-\sum_{t=1}^{l-1} \mathbf{c}_{J_{t}} \mid J_{l}=j_{l}\right], \quad j_{l} \in \mathcal{I}_{l} .
$$

Similar to the steps taken in (11), the codevectors 25 can be parameterized in terms of encoding regions, channel transition probabilities and MMSE estimation. Here, for the sake of analysis, we only provide closed-form codebook expressions for $L=2$ which are given by

$$
\begin{aligned}
\mathbf{c}_{j_{1}}^{\star} & =\frac{\sum_{i_{1}} P\left(j_{1} \mid i_{1}\right) \int_{\mathcal{R}_{i_{1}}} \widetilde{\mathbf{x}}(\mathbf{y}) f(\mathbf{y}) d \mathbf{y}}{\sum_{i_{1}} P\left(j_{1} \mid i_{1}\right) \int_{\mathcal{R}_{i_{1}}} f(\mathbf{y}) d \mathbf{y}}, \\
\mathbf{c}_{j_{2}}^{\star} & =\frac{\sum_{i_{1}, i_{2}} P\left(j_{2} \mid i_{2}\right) \int_{\mathcal{R}_{i_{1}}^{i_{2}}}\left(\widetilde{\mathbf{x}}(\mathbf{y})-\sum_{j_{1}} P\left(j_{1} \mid i_{1}\right) \mathbf{c}_{j_{1}}\right) f(\mathbf{y}) d \mathbf{y}}{\sum_{i_{1}, i_{2}} P\left(j_{2} \mid i_{2}\right) \int_{\mathcal{R}_{i_{1}}^{i_{2}}} f(\mathbf{y}) d \mathbf{y}} .
\end{aligned}
$$

Finally, we note that when $L=1$, the condition 25 simplifies into 11.

3) Training Algorithm: Similar to Algorithm 1, we can develop a practical method for training channel-optimized MSVQ for CS, coined COMSVQ-CS, summarized in Algorithm 2. Similar remarks, as stated for Algorithm 1, can be also considered for implementing Algorithm 2 with the difference that convergence in step (8) may be checked by tracking the distortion $D_{l}$, and terminate the iterations when the relative improvement is small enough. Furthermore, in order to calculate the codevector $\mathbf{c}_{j_{l}}\left(j_{l} \in \mathcal{I}_{l}\right)$ in $(25)$, we use Monte-Carlo method by first generating a set of finite training vectors $\mathbf{X}$, with known pdf, and then calculating the vector $\mathbf{X}-\sum_{t=1}^{l-1} \mathbf{c}_{J_{t}}$. Finally, we average over those vectors that have resulted the index $J_{l}=j_{l}$. 


$$
\begin{aligned}
i_{l}^{\star} & =\arg \min _{i_{l} \in \mathcal{I}_{l}} D_{l}\left(\mathbf{y}, \mathbf{i}_{1}^{l}\right) \\
& =\arg \min _{i_{l} \in \mathcal{I}_{l}}\left\{\mathbb{E}\left[\|\mathbf{X}\|_{2}^{2} \mid \mathbf{Y}=\mathbf{y}, \mathbf{I}_{1}^{l}=\mathbf{i}_{1}^{l}\right]+\mathbb{E}\left[\left\|\mathbf{c}_{J_{1}}+\ldots+\mathbf{c}_{J_{l}}\right\|_{2}^{2} \mid \mathbf{Y}=\mathbf{y}, \mathbf{I}_{1}^{l}=\mathbf{i}_{1}^{l}\right]-2 \mathbb{E}\left[\mathbf{X}^{\top}\left(\mathbf{c}_{J_{1}}+\ldots+\mathbf{c}_{J_{l}}\right) \mid \mathbf{Y}=\mathbf{y}, \mathbf{I}_{1}^{l}=\mathbf{i}_{1}^{l}\right]\right\} \\
& \stackrel{(a)}{=} \arg \min _{i_{l} \in \mathcal{I}_{l}}\left\{\mathbb{E}\left[\left\|\mathbf{c}_{J_{1}}+\ldots+\mathbf{c}_{J_{l}}\right\|_{2}^{2} \mid \mathbf{Y}=\mathbf{y}, \mathbf{I}_{1}^{l}=\mathbf{i}_{1}^{l}\right]-2 \mathbb{E}\left[\mathbf{X}^{\top}\left(\mathbf{c}_{J_{1}}+\ldots+\mathbf{c}_{J_{l}}\right) \mid \mathbf{Y}=\mathbf{y}, \mathbf{I}_{1}^{l}=\mathbf{i}_{1}^{l}\right]\right\} \\
& \stackrel{(b)}{=} \arg \min _{i_{l} \in \mathcal{I}_{l}}\left\{\mathbb{E}\left[\left\|\mathbf{c}_{J_{l}}\right\|_{2}^{2} \mid I_{l}=i_{l}\right]+2 \sum_{t=1}^{l-1} \mathbb{E}\left[\mathbf{c}_{J_{l}}^{\top} \mid I_{l}=i_{l}\right] \mathbb{E}\left[\mathbf{c}_{J_{t}} \mid I_{t}=i_{t}\right]-2 \mathbb{E}\left[\mathbf{X} \mathbf{X}^{\top} \mid \mathbf{Y}=\mathbf{y}\right] \mathbb{E}\left[\mathbf{c}_{J_{l}} \mid I_{l}=i_{l}\right]\right\}
\end{aligned}
$$

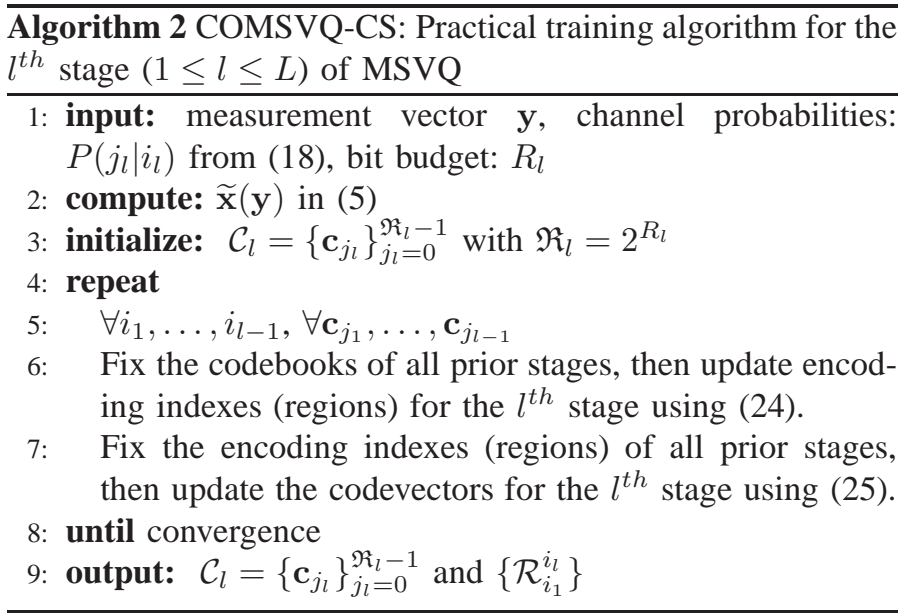

\section{Complexity of COMSVQ-CS}

In order to calculate the MSVQ encoder complexity, we calculate the number of operations at the encoder based on (24). Here, the computational complexity of CS reconstruction algorithm is not considered. We consider the argument of 24 which requires two FLOP's for the subtraction and addition, and also $2 N-1$ FLOP's for computing the second inner product term. Note that the first constant term and the third inner product term can be computed offline, and they are not counted in our complexity analysis. Thus, in total, the COMSVQ-CS encoder requires $(2 N+1) \sum_{l=1}^{L} 2^{R_{l}}$ operations, where $R_{l}(l=1, \ldots, L)$ is the quantization rate available at a $l^{\text {th }}$ stage and $L$ is total number of stages such that $\sum_{l=1}^{L} R_{l}=R$.

It can be also shown that at stage $l$, the encoder requires one float to store the first term in (24), i.e., $\left\|\mathbf{c}_{j_{l}}\right\|_{2}^{2}, N$ floats to store the second term in (24), i.e., $\mathbf{c}_{j_{l}}$, and also $l-1$ floats for storing the third term in (24). Therefore, considering $L$ stages, the total encoding memory of the COMSVQ-CS is $\sum_{l=1}^{L}(N+l) 2^{R_{l}}$. Now, we consider the decoder memory complexity. Each decoder at stage $l$ requires $N 2^{R_{l}}$ floats to store the codevector $\mathbf{c}_{j_{l}}$ considering the fact that the memory for storing the codebooks of previous stages has been already calculated. Hence, the decoder storage memory is $N \sum_{l=1}^{L} 2^{R_{l}}$ floats.

By splitting the original VQ into stages, the computational complexity as well as memory complexity can be considerably reduced. Therefore, a practical, however sub-optimal, implementation of COVQ-CS is feasible at high quantization rate and dimension.

\section{EXPERIMENTS AND RESULTS}

In this section, we evaluate the performance of the proposed designs COVQ-CS (Algorithm 1) and COMSVQ-CS (Algorithm 2). Through simulations, we compare their performances with the lower-bounds developed in Section III-D along with existing quantizers used for CS. We consider three quantizers following the system model of Figure 3. They are as follows.

- Nearest-Neighbor Coding for CS (NNC-CS) 11 The NNCCS design method has been discussed in Section IV-B. Note that this scheme has the same complexity order as that of the COVQ-CS.

- Multi-Stage Nearest-Neighbor Coding for CS (MSNNC$C S$ ): Using multi-stage structure for NNC-CS leads to the design of MSNNC-CS. The quantizer encoding-decoding conditions using this design are given in [32] for a non-CS system model. The encoding complexity order of MSNNC$\mathrm{CS}$ is the same as that of the COMSVQ-CS.

- Basis Pursuit DeQuantizing (BPDQ) [9]: Using this method, the encoder uniformly scalar-quantizes CS measurements, and the BPDQ algorithm [9] reconstructs the sparse source (from the quantized measurements) by solving the following convex optimization problem

$$
\mathbf{x}^{\star}=\arg \min _{\mathbf{x} \in \mathbb{R}^{N}}\|\mathbf{x}\|_{1} \text { s.t. }\|\widehat{\mathbf{y}}-\mathbf{\Phi} \mathbf{x}\|_{p} \leq \gamma,
$$

where $\widehat{\mathbf{y}}$ is the quantized vector, $p>2$ and $\gamma>0$ is chosen to satisfy some fidelity constraint, e.g., quantization error power. Note that the encoder computational complexity is of order $\mathcal{O}\left(2^{R / M}\right)$. In the design of uniform scalar quantizer for the BPDQ scheme, the choice of lower- and upper-boundaries for quantization is important, leading to different saturation errors [33]. In order to choose the end-points for uniform quantization of CS measurements, we generate random samples of CS measurement vectors according to the distribution of the sparse source, sensing matrix, and the measurements noise. Then, the upper quantization boundary is selected as the maximum value among the amplitudes of the generated sample entries of the measurement vector. The lower quantization boundary is also selected as the opposite value of the upper-boundary. Using such simple approach, we mainly reduce the effect of the saturation error.

The following scheme follows the system model of Figure 2

\footnotetext{
${ }^{1}$ Here, with abuse of notation we use the term nearest-neighbor coding in the presence of channel noise instead of weighted nearest-neighbor coding.
} 
- Support Set Coding (SSC): In the SSC method, the reconstructed support set of $\widetilde{\mathbf{x}}(\mathbf{y})$ is transmitted using $\log _{2}\left(\begin{array}{l}N \\ K\end{array}\right)$ bits, and then the $K$ largest coefficients (in magnitude) within the reconstructed support set are scalar-quantized to their nearest neighbor codepoints using $R-\log _{2}\left(\begin{array}{l}N \\ K\end{array}\right)$ bits. Here, we use codepoints optimized for a standard Gaussian RV using the LBG algorithm [30]. Notice that when the non-zero coefficients of the sparse source vector are drawn according to an i.i.d. standard Gaussian distribution (which is the case in our simulations), the optimized LBG-based codepoints minimize the distortion per nonzero component of the sparse source. It is straightforward that the encoding complexity of the SSC is of order $\mathcal{O}\left(2^{\left(R-\log _{2}\left(\begin{array}{l}N \\ K\end{array}\right)\right) / K}\right)$, or equivalently $\mathcal{O}\left(2^{R / K}\right)$ at high quantization rate. 1

\section{A. Experimental Setup}

We quantify the performance using normalized MSE (NMSE) defined as

$$
\mathrm{NMSE} \triangleq \frac{\mathbb{E}\left[\|\mathbf{X}-\widehat{\mathbf{X}}\|_{2}^{2}\right]}{\mathbb{E}\left[\|\mathbf{X}\|_{2}^{2}\right]}
$$

In principle, the numerator of NMSE in 27) is computed by sample averaging over generated realizations of $\mathbf{X}$ using MonteCarlo simulations, and the denominator can be calculated exactly under the assumptions of our simulation setup.

In addition, in order to measure the level of under-sampling, we define the measurement rate $0<\alpha \leq 1$ as $\alpha \triangleq M / N$.

Our simulation setup includes the following steps:

1) For given values of sparsity level $K$ (assumed known in advance) and input vector size $N$, choose $\alpha$, and round the number of measurements $M$ to its nearest integer.

2) Randomly generate a set of exactly $K$-sparse vector $\mathbf{X}$, where the support set $\mathcal{S}$ with $|\mathcal{S}|=K$ is chosen uniformly at random over the set $\{1,2, \ldots, N\}$. The non-zero coefficients of $\mathbf{X}$ are i.i.d. and drawn from standard Gaussian source $\mathcal{N}(0,1)$; Hence $\mathbb{E}\left[\|\mathbf{X}\|_{2}^{2}\right]=K$.

3) We let the elements of the sensing matrix be $\boldsymbol{\Phi}_{i j} \stackrel{\text { iid }}{\sim}$ $\mathcal{N}(0,1 / M)$, and normalize its columns to unit-norm. Once $\Phi$ is generated, it remains fixed and known globally.

4) Compute linear measurements $\mathbf{Y}=\mathbf{\Phi} \mathbf{X}+\mathbf{W}$ for each sparse data vector where $\mathbf{W} \sim \mathcal{N}\left(\mathbf{0}, \sigma_{w}^{2} \mathbf{I}_{M}\right)$.

5) We Choose the total quantization rate $R$, and assume a BSC with bit cross-over probability $0 \leq \epsilon \leq 0.5$ specified by

$$
P(k \mid l)=\epsilon^{H_{\mathfrak{R}}(k, l)}(1-\epsilon)^{\mathfrak{R}-H_{\mathfrak{R}}(k, l)}, \mathfrak{R}=2^{R},
$$

where $0 \leq \epsilon \leq 1 / 2$ represents bit cross-over probability (assumed known), and $H_{\mathfrak{R}}(k, l)$ denotes the Hamming distance between $\mathfrak{R}$-bit binary codewords representing the channel input and output indexes $k$ and $l$. The capacity of BSC (in bits per channel use) with bit cross-over probability $\epsilon$ is equivalent to

$$
C=1+\epsilon \log _{2}(\epsilon)+(1-\epsilon) \log _{2}(1-\epsilon) .
$$

\footnotetext{
${ }^{1}$ In the spirit of reproducible results, we provide MATLAB codes for simulation of the AbS-based quantizers in the following website: www.ee.kth.se/ amishi/reproducible_research.html.
}

6) Apply the quantization algorithms on the generated data $\mathbf{Y}$, and assess NMSE by averaging over all data.

7) Practical necessity: In our proposed COVQ-CS and COMSVQ-CS design algorithms, it is required to calculate the MMSE estimator $\widetilde{\mathbf{x}}(\mathbf{y})$, e.g. in (8) and (24). Implementing the Bayesian MMSE estimator, or in other words, calculating the conditional mean $\mathbb{E}[\mathbf{X} \mid \mathbf{Y}=\mathbf{y}]$, has been studied in [20]-[24] which can be derived approximately or exactly under certain assumptions. Although the MMSE estimator can be implemented for low-dimensional vectors (as used in Example 2), as the dimension grows, its complexity increases exponentially. Thus, for the sake of complexity, we will approximate $\widetilde{\mathbf{x}}(\mathbf{y})$ using the output of a practically realizable CS reconstruction algorithm. Considering the case that a $\ell_{1}$-norm minimization-based convex reconstruction also suffers from high complexity $\mathcal{O}\left(N^{3}\right)$ for a high dimension $N$, we choose the simple orthogonal matching pursuit (OMP) greedy algorithm [34] as a CS reconstruction where its computational complexity is $\mathcal{O}\left(K^{3}+K^{2} M+K M N\right)$. We show that using the OMP algorithm, we can obtain reasonable performance. The OMP is used as the approximation of the MMSE estimator (at the encoder side) for COVQ-CS, COMSVQ$\mathrm{CS}$ and SSC schemes as well as the realization of CS reconstruction algorithm (at the decoder side) for NNCCS and MSNNC-CS methods.

\section{B. Experimental Results}

In our simulations, we generate $10^{6}$ realizations of the input sparse vector $\mathbf{X}$ (correspondingly $\mathbf{Y}$ ) for the training algorithms as well as performance assessments using Monte-Carlo simulations. We evaluate the performance of the competing schemes in terms of number of CS measurements $(\alpha)$, total quantization rate $(R)$ and channel condition $(\epsilon)$. It should be also mentioned that the training algorithms are performed at each value on $\mathrm{x}$ axis, i.e., $\alpha, R$ and $\epsilon$.

In our first experiment, we assume that the measurement noise and channel cross-over probability are negligible, i.e., $\sigma_{w}^{2}=0$ and $\epsilon=0$. 2 In Figure 7 with the simulation setup ( $N=12, K=2, R=12$ bits/vector), we vary measurement rate $\alpha=M / N$, and compare the performance (NMSE) of the quantizers along with the lower-bound (14). We use 2stage VQ with equal quantization rates. For implementing the BPDQ decoder, we select $p=3$ in (26) (the choice of $p$ is experimentally verified to achieve the best performance), and $\gamma$ is chosen according to [9, eq. (7)], then a standard convex solver is used to find the optimal solution of 26). Let us first investigate the behavior of the full search COVQ-CS and NNC-CS quantizer design schemes in Figure 7 At a fixed quantization rate $R$, increasing the number of measurements improves the CS reconstruction performance, hence the endto-end MSE decreases. Since quantized transmission distortion $D_{q}$ is fixed, NMSE would saturate ultimately. As expected, the proposed COVQ-CS design method gives the best performance, and at high measurement rates, it approaches the lower-bound

\footnotetext{
${ }^{2}$ With abuse of notation, we still use the term COVQ-CS and COMSVQ-CS when channel is noiseless $(\epsilon=0)$.
} 


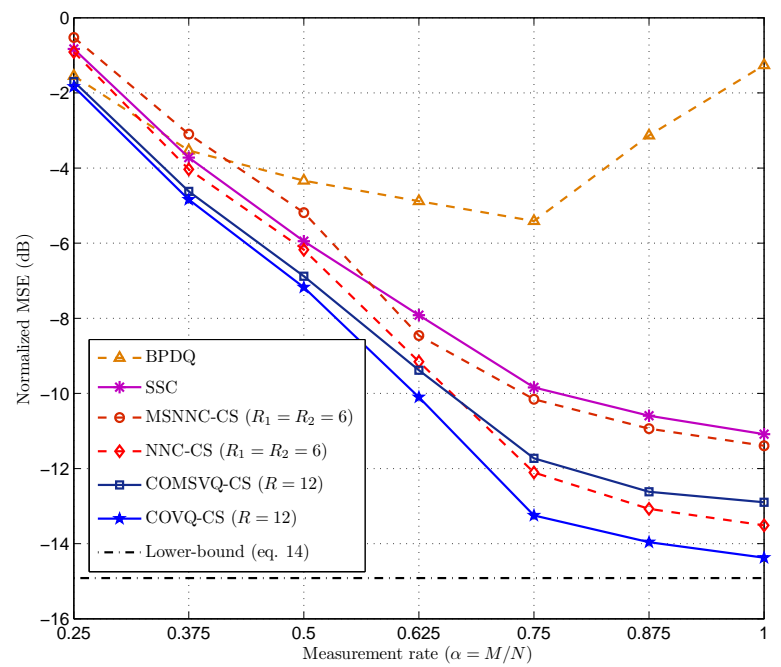

Fig. 7. NMSE (in $\mathrm{dB}$ ) as a function of measurement rate $\alpha=M / N$ using different quantizer design schemes. The parameters are chosen as $N=12$, $K=2$ and $R=12$ bits/vector for a noiseless channel and clean measurements.

(14). This is due to the fact that, at high measurement rate regime, the CS distortion $D_{c s}$ becomes negligible and the source vector can be precisely recovered from the measurements; therefore, COVQ-CS approaches the distortion rate function for the sparse source. Note that the performance gain using the COVQ-CS design scheme is obtained at the expense of computational and memory complexity of order $\mathcal{O}\left(2^{R}\right)$. Using the sub-optimal COMSVQ-CS scheme, the complexity is decreased to the order $\mathcal{O}\left(2^{R / 2}\right)$ although its performance is slightly declined compared to COVQ-CS. Among multistage structured methods, the COMSVQ-CS performs better than MSNNC-CS since it takes end-to-end MSE through its design procedure. Also, it can closely follow the behavior of the COVQ-CS at low to moderate ranges of measurement rates since at this regime, the performance is mostly influenced by the CS reconstruction distortion. However, as the measurement rate increases, the gap between the performance of the COMSVQCS and COVQ-CS becomes larger. The gap can be made smaller if we use higher quantization rates at the first stage while keeping the total quantization rate fixed, however, this imposes more encoding complexity to the system. It can be also seen that the SSC scheme performs poorer than COVQ-CS and MSVQ-CS, while its encoding complexity grows at most like $O\left(2^{R / 2}\right)$. The behavior of the BPDQ, however, is different: increasing number of measurements, on one hand, facilitates a more precise reconstruction. On the other hand, it reduces quantization rate since each measurement entry is quantized using $R / M$ bits. Hence, the performance curve of BPDQ reaches a minimum point, and then takes an upward trend which also complies with the fact of CS and quantization compression regimes [18]. Note that the BPDQ has the least computational complexity among the competing techniques varying from the order of $\mathcal{O}\left(2^{R / 3}\right)$ to $\mathcal{O}\left(2^{R / 12}\right)$.

In our next experiment, we use larger dimension and quantization rate as $(N=32, K=3, M=20(\alpha=0.625)$,

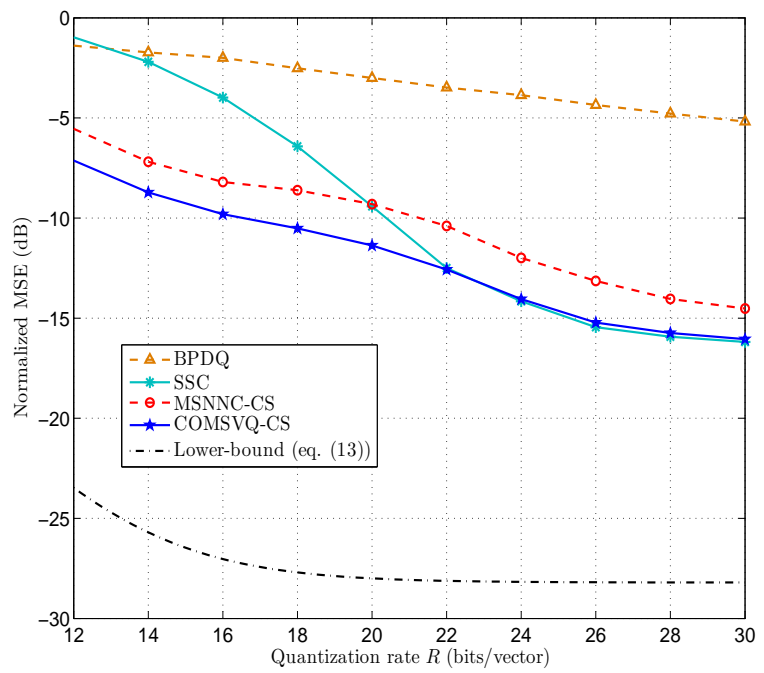

Fig. 8. NMSE (in $\mathrm{dB}$ ) as a function of total quantization rate $R$ (in bits/vector). Simulation parameters are chosen as $N=32, K=3, M=20$ for noiseless channel and $\sigma_{w}^{2}=0.005$.

$\left.\sigma_{w}^{2}=0.005\right)$ and noiseless channel. In Figure 8 we plot the NMSE of low-complexity quantizer designs, i.e., COMSVQCS, MSNNC-CS, SSC and BPDQ, along with the lowerbound (13) by varying total quantization rate $R$. The mutual coherence $\mu$ in the lower-bound (13) is computed by (2) (here, $\mu=0.5755$ ). For implementing multi-stage quantizers, we assume two stages with $R_{1}=R_{2}$. Also, BPDQ parameters are the same as those of the previous simulation study. At low to moderate quantization rate regimes, the COMSVQCS outperforms other techniques; for example, at $R=20$ bits/vector, it has almost $2 \mathrm{~dB}$ performance gain over MSNNC$\mathrm{CS}$ and SSC, and $8 \mathrm{~dB}$ performance gain over BPDQ. The performance of SSC differs much at low to high quantization rates: although its performance is very poor at low to moderate rates, the performance reaches that of the COMSVQ-CS at high rates since the SSC requires high rates to perform well. Note that, at low quantization rates, the performance of SSC is poor due to the reason that its design is based on scalar quantization of reconstructed source vector at the encoder. Whereas, the COMSVQ-CS and MSNNC-CS schemes provide better performance since, in their designs, reconstructed source vector or CS measurement vector are vector-quantized. Naturally, this performance gain is achieved at the expense of higher encoding complexity. Note that all schemes attain a MSE floor ultimately due to the additive noise which is reflected from the lowerbound as well. In particular, at very high quantization rates, the performance of SSC approaches to that of the COMSVQ-CS, and finally converges to the CS reconstruction MSE, denoted by $D_{c s}$, which is also aligned with our findings in Remark 3 . Our calculations show that the MSE floor, i.e., the value of $D_{c s}$, is approximately $-16.5 \mathrm{~dB}$.

In our final experiments, we consider the effect of channel noise on the performance of the proposed JSCC schemes, and we also compare them with separate source-channel coding 
schemes. In Figure 9 and Figure 10, we quantify the performance as a function of channel bit cross-over probability $\epsilon$, respectively, for two parameter sets: $(N=12, K=2, M=9$ ( $\alpha=$ $0.75), R=15$ bits/vector, $\left.\sigma_{w}^{2}=0\right)$ and $(N=32, K=3, M=20$ ( $\alpha=0.625), R=20$ bits/vector, $\sigma_{w}^{2}=0$ ). In Figure 9 , we observe that the proposed designs, i.e., COVQ-CS and COMSVQ-CS (with $R_{1}=8$ and $R_{2}=7$ bits/vector) always outperform other schemes. The curves labeled by 'SSC-BCH' and 'BPDQ-BCH', respectively, consist of twelve-dimensional 11-bit encoded bits using SSC and uniform quantization, followed by $(15,11) \mathrm{BCH}$ codes (this rate is experimentally tested to obtain the best performance among $\mathrm{BCH}$ rate allocations.). Note that channel coding rates are chosen in order to have a fair comparison (in terms of same delay) among JSCC schemes (COVQ-CS and COMSVQ-CS) and separate source-channel coding methods (SSC-BCH and BPDQ-BCH). We observe from Figure 9 that using separate channel coding, the performance of the BPDQ is still poor. It can be also seen that the SSC, even equipped with channel coding, is highly susceptible to channel noise since an error in receiving the support set may detrimentally degrade the performance. Therefore, the MSE increases more rapidly as compared to COVQ-CS, COMSVQ-CS and BPDQ$\mathrm{BCH}$. We have also tested the performance of SSC with $(15,11)$ Hamming codes which provides almost the same performance as that of the SSC-BCH. Using joint source-channel codes in the proposed designs enhances the performance and provide robustness, particularly at high channel noise. For example, in Figure 9 the performance gain of COVQ-CS over the SSC is almost $4 \mathrm{~dB}$, when the channel is highly noisy $(\epsilon=0.05)$. While the COMSVQ-CS and SSC have (almost) the same encoding complexity order, the performance gain of COMSVQ-CS over SSC-BCH is more than $2 \mathrm{~dB}$ at $\epsilon=0.05$. It should be mentioned that the gap between the COVQ-CS and the lower-bound is due to CS reconstruction distortion (low number of measurements) as well as finite length of source-channel codes.

Since the SSC is quite sensitive to error in received support set, in Figure 10, we also show the performance of SSC when the reconstructed support set is transmitted without loss, and the non-zero coefficients are encoded using $(7,4) \mathrm{BCH}$ codes. This scheme is marked by 'SSC-coded' in Figure 10, and, indeed, is an ideal coding scheme since the support set may not be transmitted losslessly over a noisy channel, in practice. The proposed COMSVQ-CS design method not only outperforms the ideal separate source-channel coding scheme (SSC-coded), but also the other JSCC scheme (MSNNC-CS). It can be also seen as channel condition degrades, the COMSVQ-CS curve increases with the same slope as that of the lower-bound.

As a final remark, we mention that the $\mathrm{VQ}$, in general, is known to be theoretically the optimal block coding strategy. However, its computational and memory complexity is an issue, which has been addressed using multi-stage VQ in the current work. In our simulations, the implementation of COVQ-CS and COMSVQ-CS (using two stages) might not be performed beyond $R=12$ bits/vector for a dimension $N=12$ (or slightly more), and $R=30$ for a dimension $N=32$, respectively. If an implementation of a VQ for CS measurements of sparse sources with close to real-life dimensions (e.g., $N=256$ or even higher) is desired, one needs to consider a VQ with multiple

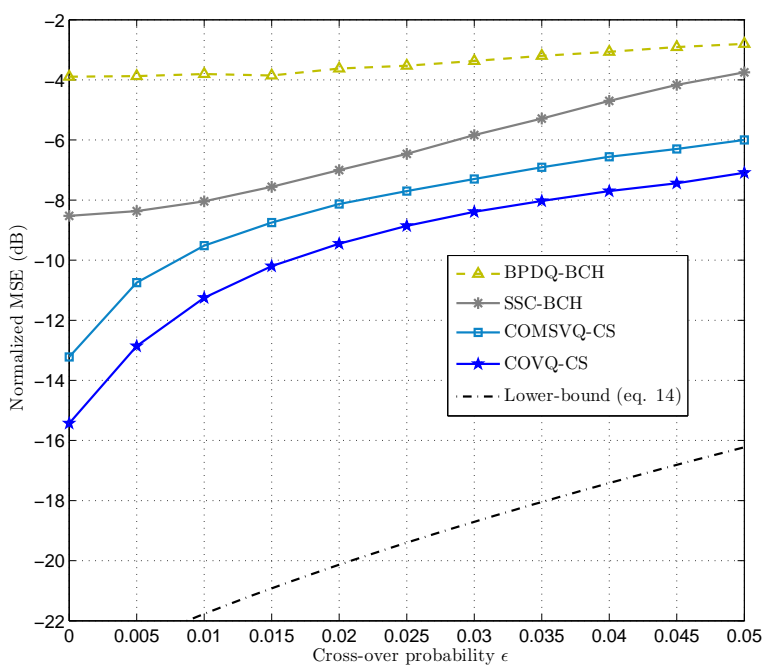

Fig. 9. NMSE (in $\mathrm{dB}$ ) as a function of channel bit cross-over probability $\epsilon$. Simulation parameters are chosen as $N=12, K=2, M=9$ and $R=15$ bits/vector for clean CS measurements.

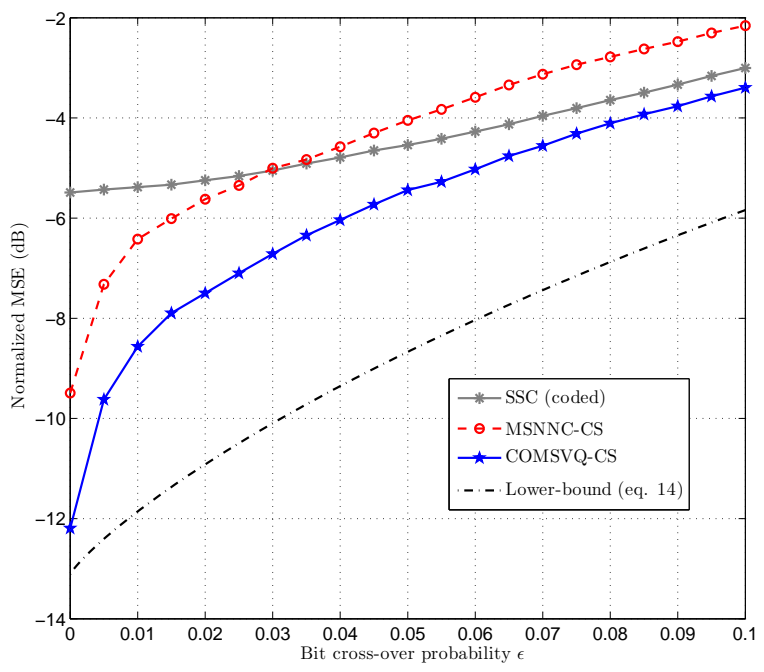

Fig. 10. NMSE (in $\mathrm{dB}$ ) as a function of channel bit cross-over probability $\epsilon$. Simulation parameters are chosen as $N=32, K=3, M=20$ and $R=20$ bits/vector for clean CS measurements.

stages (more than two). Another alternative is to use VQ for a high-dimensional source vector by segmenting the source into different small patches of information, and use the segmented patches for training 1 This approach, for example, is used in vector quantization of images [31, Chapter 11] or in image denoising [35].

\section{CONCLUSION}

We have developed optimum joint source-channel vector quantization schemes for CS measurements. We have derived

\footnotetext{
${ }^{1}$ In the context of this paper, the source can be thought of as the sparse representation (e.g., wavelet coefficients, etc.) of an image.
} 
necessary conditions for optimality of VQ encoder-decoder pair with respect to end-to-end MSE. One interesting result of the optimal conditions is that the CS reconstruction should be performed MMSE-wise at the encoder side rather than the decoder side. We have also provided a theoretical lower-bound on the MSE performance based on the fact that the end-toend MSE can be decomposed into CS reconstruction MSE and quantized transmission MSE without loss of optimality. Using the resulting optimal conditions, we have proposed a practical encoder-decoder design through an iterative algorithm referred to as COVQ-CS. Moreover, the encoding complexity of VQ was addressed using the MSVQ where we have approximated the necessary optimal conditions by applying a multi-stage structure which has led to the design of COMSVQ-CS. Numerical results show promising performance of the proposed designs with respect to relevant methods in literature.

\section{APPENDIX}

Note that the end-to-end MSE, $\mathbb{E}\left[\|\mathbf{X}-\widehat{\mathbf{X}}\|_{2}^{2}\right]$, of the system of Figure 1 is always larger than the MSE of a system with a priori known (oracle) support set $\mathcal{S}$ under the same assumptions. Let the RV's $\left.\mathbf{X}\right|_{\mathcal{S}},\left.\widetilde{\mathbf{X}}\right|_{\mathcal{S}},\left.\widehat{\mathbf{X}}\right|_{\mathcal{S}} \in \mathbb{R}^{N}$, respectively, denote the source vector, the MMSE estimation of the source given measurements, and the final reconstruction vector given the known support set $\mathcal{S}$. Therefore, we have

$$
\begin{aligned}
\mathbb{E}\left[\|\mathbf{X}-\widehat{\mathbf{X}}\|_{2}^{2}\right] & \geq \mathbb{E}\left[\left\|\left.\mathbf{X}\right|_{\mathcal{S}}-\left.\widehat{\mathbf{X}}\right|_{\mathcal{S}}\right\|_{2}^{2}\right] \\
& =\mathbb{E}\left[\left\|\left.\mathbf{X}\right|_{\mathcal{S}}-\left.\widetilde{\mathbf{X}}\right|_{\mathcal{S}}\right\|_{2}^{2}\right]+\mathbb{E}\left[\left\|\left.\widetilde{\mathbf{X}}\right|_{\mathcal{S}}-\left.\widehat{\mathbf{X}}\right|_{\mathcal{S}}\right\|_{2}^{2}\right],
\end{aligned}
$$

where, the equality in 30 follows from the same reasoning as that of (12) $)^{2}$ Let us first develop a lower-bound on $\mathbb{E}\left[\|\left.\mathbf{X}\right|_{\mathcal{S}}-\right.$ $\left.\left.\widetilde{\mathbf{X}}\right|_{\mathcal{S}} \|_{2}^{2}\right]$. Defining $\boldsymbol{\Phi}_{\mathcal{S}} \in \mathbb{R}^{M \times K}$ as a sub-matrix of $\boldsymbol{\Phi}$ formed by choosing its columns indexed by the elements of $\mathcal{S}$, then for a single realization of $\mathcal{S}$, we have

$$
\begin{aligned}
& \mathbb{E}\left[\left\|\left.\mathbf{X}\right|_{\mathcal{S}}-\left.\widetilde{\mathbf{X}}\right|_{\mathcal{S}}\right\|_{2}^{2}\right] \stackrel{(a)}{=} \operatorname{Tr}\left\{\left(\mathbf{I}_{K}+\frac{1}{\sigma_{w}^{2}} \boldsymbol{\Phi}_{\mathcal{S}}^{\top} \boldsymbol{\Phi}_{\mathcal{S}}\right)^{-1}\right\} \\
& \stackrel{(b)}{\geq} \frac{K^{2}}{K+\frac{1}{\sigma_{w}^{2}} \operatorname{Tr}\left\{\boldsymbol{\Phi}_{\mathcal{S}}^{\top} \boldsymbol{\Phi}_{\mathcal{S}}\right\}} \geq \frac{K}{1+\frac{1}{\sigma_{w}^{2}} \lambda_{\max }\left\{\boldsymbol{\Phi}_{\mathcal{S}}^{\top} \boldsymbol{\Phi}_{\mathcal{S}}\right\}} \\
& \stackrel{(c)}{\geq} \frac{K}{1+\frac{1}{\sigma_{w}^{2}}(1+(K+1) \mu)},
\end{aligned}
$$

where in $(a)$, we use the MMSE estimation error of a Gaussian source $\mathbf{X}_{\mathcal{S}} \sim \mathcal{N}\left(\mathbf{0}, \mathbf{I}_{K}\right)$ (elements of $\left.\mathbf{X}\right|_{\mathcal{S}}$ within the support set) in white Gaussian measurement noise $\mathbf{W} \sim \mathcal{N}\left(\mathbf{0}, \sigma_{w}^{2} \mathbf{I}_{M}\right)$ (see [26, Theorem 11.1] for details). Also, (b) follows from the fact that for a given positive-definite matrix $\mathbf{B} \in \mathbb{R}^{K \times K}$, $\operatorname{Tr}\{\mathbf{B}\} \operatorname{Tr}\left\{\mathbf{B}^{-1}\right\} \geq K^{2}$ which can be shown using the CauchySchwarz inequality (see e.g. [36, Lemma 2]). Further, (c) holds since all eigenvalues of $\boldsymbol{\Phi}_{\mathcal{S}}^{\top} \boldsymbol{\Phi}_{\mathcal{S}}$ are upper-bounded by $1+(K+$ 1) $\mu$ using Gershgorin disc theorem [37, Theorem 8.1.3]. Now, since the oracle support set is drawn uniformly at random from all $\left(\begin{array}{l}N \\ K\end{array}\right)$ possibilities, the final inequality in 31 is the lowerbound for all realizations of $\mathcal{S}$.

\footnotetext{
${ }^{2}$ We drop the dependency of $\left.\widetilde{\mathbf{X}}\right|_{\mathcal{S}}$ on $\mathbf{Y}$ for simplicity of notation.
}

Next, we develop a lower-bound on the quantized transmission distortion $\mathbb{E}\left[\left\|\left.\widetilde{\mathbf{X}}\right|_{\mathcal{S}}-\left.\widehat{\mathbf{X}}\right|_{\mathcal{S}}\right\|_{2}^{2}\right]$. It should be noted that the elements of $\left.\widetilde{\mathbf{X}}\right|_{\mathcal{S}}$ within the known support set, denoted by $\widetilde{\mathbf{X}}_{\mathcal{S}} \in \mathbb{R}^{K}$, are Gaussian with the covariance matrix (see $[26$ Theorem 10.3])

$$
\operatorname{cov}\left[\widetilde{\mathbf{X}}_{\mathcal{S}}\right]=\left(\mathbf{I}_{K}+\frac{1}{\sigma_{w}^{2}} \boldsymbol{\Phi}_{\mathcal{S}}^{\top} \boldsymbol{\Phi}_{\mathcal{S}}\right)^{-1}
$$

In order to find the minimum distortion, or distortion-rate function, caused by quantization of a sparse source $\left.\widetilde{\mathbf{X}}\right|_{\mathcal{S}}$, a natural approach is to let the quantizer encoder first encode the support set elements using $\log _{2}\left(\begin{array}{l}N \\ K\end{array}\right)$ bits (since the elements of $\mathcal{S}$ are drawn uniformly) which can be received without loss at the decoder, and then encode the correlated Gaussian vector $\widetilde{\mathbf{X}}_{\mathcal{S}}$ using $R-\log _{2}\left(\begin{array}{l}N \\ K\end{array}\right)$ bits. It is shown in [27] that the distortion rate function of this splitting approach coincides with the distortion-rate function for a sparse source (with Gaussian non-zero coefficients and uniformly distributed sparsity pattern) asymptotically (in quantization rate $R$ with $R \gg \log _{2}\left(\begin{array}{l}N \\ K\end{array}\right)$ ). Then, it follows that

$\mathbb{E}\left[\left\|\left.\widetilde{\mathbf{X}}\right|_{\mathcal{S}}-\left.\widehat{\mathbf{X}}\right|_{\mathcal{S}}\right\|_{2}^{2}\right] \geq c 2^{-2 C\left(\frac{R-\log _{2}\left(\begin{array}{c}N \\ K\end{array}\right)}{K}\right)} \operatorname{det}\left(\operatorname{cov}\left[\widetilde{\mathbf{X}}_{\mathcal{S}}\right]\right)^{\frac{1}{K}}$

where $c=2\left(\frac{K}{2} \Gamma\left(\frac{K}{2}\right)\right)^{\frac{2}{K}}\left(\frac{K+2}{K}\right)^{\frac{K}{2}}$. The right-hand side in (33) is indeed the distortion-rate function of the correlated Gaussian source $\widetilde{\mathbf{X}}_{\mathcal{S}}$ [38] incurred by transmission over the DMC with capacity $C$ (see, e.g. source-channel separation theorem [28, Chapter 7]). Further, we have

$$
\begin{aligned}
& \operatorname{det}\left(\operatorname{cov}\left[\tilde{\mathbf{X}}_{\mathcal{S}}\right]\right)^{\frac{1}{K}}=\frac{1}{\prod_{k=1}^{K}\left(1+\frac{1}{\sigma_{w}^{2}} \lambda_{k}\left(\boldsymbol{\Phi}_{\mathcal{S}}^{\top} \boldsymbol{\Phi}_{\mathcal{S}}\right)\right)^{\frac{1}{K}}} \\
& \geq \frac{1}{1+\frac{1}{\sigma_{w}^{2}} \lambda_{\max }\left(\boldsymbol{\Phi}_{\mathcal{S}}^{\top} \boldsymbol{\Phi}_{\mathcal{S}}\right)} \stackrel{(a)}{\geq} \frac{1}{1+\frac{1}{\sigma_{w}^{2}}(1+(K+1) \mu)},
\end{aligned}
$$

where in $(a)$ we use the fact that all eigenvalues of $\boldsymbol{\Phi}_{\mathcal{S}}^{\top} \boldsymbol{\Phi}_{\mathcal{S}}$ are upper-bounded by $1+(K+1) \mu$ using Gershgorin disc theorem. Combining (34) with 33, 31) and 30 concludes the proof.

\section{REFERENCES}

[1] A. Shirazinia, S. Chatterjee, and M. Skoglund, "Channel-optimized vector quantizer design for compressed sensing measurements," in Proc. IEEE International Conference on Acoustics, Speech and Signal Processing (ICASSP), May 2013, pp. 4648-4652.

[2] E. Candes and M. Wakin, "An introduction to compressive sampling," IEEE Signal Processing Magazine, vol. 25, no. 2, pp. 21 -30, Mar. 2008.

[3] J. Sun and V. Goyal, "Optimal quantization of random measurements in compressed sensing," in IEEE Int. Symp. Inf. Theory, Jul. 2009, pp. 6 -10 .

[4] U. Kamilov, V. Goyal, and S. Rangan, "Optimal quantization for compressive sensing under message passing reconstruction," in IEEE Int. Symp. Inf. Theory, 31 2011-Aug. 5 2011, pp. $459-463$.

[5] A. Shirazinia, S. Chatterjee, and M. Skoglund, "Analysis-by-synthesis quantization for compressed sensing measurements," IEEE Trans. Sig. Proc., vol. 61, no. 22, pp. 5789-5800, November 2013.

[6] C. Güntürk, M. Lammers, A. Powell, R. Saab, and O. Ylmaz, "Sigma delta quantization for compressed sensing," in Annual Conference Inf. Sciences and Systems, March 2010, pp. $1-6$.

[7] A. Zymnis, S. Boyd, and E. Candes, "Compressed sensing with quantized measurements," IEEE Sig. Proc. Lett., vol. 17, no. 2, pp. 149 -152, Feb. 2010. 
[8] W. Dai and O. Milenkovic, "Information theoretical and algorithmic approaches to quantized compressive sensing," IEEE Trans. Commun., vol. 59, no. 7, pp. $1857-1866$, Jul. 2011.

[9] L. Jacques, D. Hammond, and J. Fadili, "Dequantizing compressed sensing: When oversampling and non-Gaussian constraints combine," IEEE Trans. Inf. Theory, vol. 57, no. 1, pp. 559 -571, Jan. 2011.

[10] A. Shirazinia, S. Chatterjee, and M. Skoglund, "Performance bounds for vector quantized compressive sensing," in Int. Symp. Inf. Theory and App., Oct. 2012 , pp. $289-293$.

[11] U. S. Kamilov, V. K. Goyal, and S. Rangan, "Message-passing dequantization with applications to compressed sensing," IEEE Trans. Sig. Proc., vol. 60, no. 12, pp. 6270 -6281, Dec. 2012.

[12] P. Boufounos and R. Baraniuk, "1-bit compressive sensing," in Annual Conference Inf. Sciences and Systems, 2008, pp. 16-21.

[13] M. Yan, Y. Yang, and S. Osher, "Robust 1-bit compressive sensing using adaptive outlier pursuit," IEEE Trans. Sig. Proc., vol. 60, no. 7, pp. 3868 -3875 , Jul. 2012.

[14] L. Jacques, J. Laska, P. Boufounos, and R. Baraniuk, "Robust 1-bit compressive sensing via binary stable embeddings of sparse vectors," IEEE Trans. Inf. Theory, vol. 59, no. 4, pp. 2082-2102, 2013.

[15] Y. Plan and R. Vershynin, "Robust 1-bit compressed sensing and sparse logistic regression: A convex programming approach," IEEE Trans. Inf. Theory, vol. 59, no. 1, pp. 482-494, 2013.

[16] R. J. Pai, "Nonadaptive lossy encoding of sparse signals," Master's thesis, MIT EECS, Cambridge, August 2006.

[17] V. Goyal, A. Fletcher, and S. Rangan, "Compressive sampling and lossy compression," IEEE Sig. Proc. Mag., vol. 25, no. 2, pp. 48-56, March 2008.

[18] J. Laska and R. Baraniuk, "Regime change: Bit-depth versus measurement-rate in compressive sensing," IEEE Trans. Sig. Proc., vol. 60 , no. 7 , pp. $3496-3505$, Jul. 2012

[19] D. Donoho and X. Huo, "Uncertainty principles and ideal atomic decomposition," IEEE Trans. Inf. Theory, vol. 47, no. 7, pp. 2845 -2862, Nov 2001.

[20] E. G. Larsson and Y. Selen, "Linear regression with a sparse parameter vector," IEEE Trans. Sig. Proc., vol. 55, no. 2, pp. 451 -460, Feb. 2007.

[21] S. Ji, Y. Xue, and L. Carin, "Bayesian compressive sensing," IEEE Trans. Sig. Proc., vol. 56, no. 6, pp. 2346 -2356, June 2008

[22] M. Elad and I. Yavneh, "A plurality of sparse representations is better than the sparsest one alone," IEEE Trans. Inf. Theory, vol. 55, no. 10, pp. $4701-4714$, Oct. 2009.

[23] M. Protter, I. Yavneh, and M. Elad, "Closed-form MMSE estimation for signal denoising under sparse representation modeling over a unitary dictionary," IEEE Trans. Sig. Proc., vol. 58, no. 7, pp. 3471 -3484, Jul. 2010.

[24] K. Qiu and A. Dogandzic, "Sparse signal reconstruction via ECME hard thresholding," IEEE Trans. Sig. Proc., vol. 60, no. 9, pp. $4551-4569$, Sep. 2012.

[25] N. Farvardin and V. Vaishampayan, "On the performance and complexity of channel-optimized vector quantizers," IEEE Trans. Inf. Theory, vol. 37, no. 1, pp. $155-160$, Jan. 1991.

[26] S. Kay, Fundamentals of Statistical Signal Processing: Estimation Theory. Englewood Cliffs, NJ: Prentice Hall, 1993.

[27] C. Weidmann and M. Vetterli, "Rate distortion behavior of sparse sources," IEEE Trans. Inf. Theory, vol. 58, no. 8, pp. 4969 -4992, Aug. 2012.

[28] T. M. Cover and J. A. Thomas, Elements of Information Theory, 2nd ed. John Wiley and Sons, 2006.

[29] N. Farvardin, "A study of vector quantization for noisy channels," IEEE Trans. Inf. Theory, vol. 36, no. 4, pp. 799 -809, Jul. 1990.

[30] Y. Linde, A. Buzo, and R. Gray, "An algorithm for vector quantizer design," IEEE Trans. Commun., vol. 28, no. 1, pp. 84 - 95, Jan 1980.

[31] A. Gersho and R. M. Gray, Vector Quantization and Signal Compression. Kluwer Academic Publishers, 1991.

[32] N. Phamdo, N. Farvardin, and T. Moriya, "A unified approach to treestructured and multistage vector quantization for noisy channels," IEEE Trans. Inf. Theory, vol. 39, no. 3, pp. 835 -850, May 1993.

[33] J. N. Laska, P. T. Boufounos, M. A. Davenport, and R. G. Baraniuk, "Democracy in action: Quantization, saturation, and compressive sensing," Applied and Computational Harmonic Analysis, vol. 31, no. 3, pp. 429443, 2011.

[34] J. Tropp and A. Gilbert, "Signal recovery from random measurements via orthogonal matching pursuit," IEEE Trans. Inf. Theory, vol. 53, no. 12, pp. $4655-4666$, Dec. 2007.

[35] J. S. Turek, I. Yavneh, and M. Elad, "On MAP and MMSE estimators for the co-sparse analysis model," Digital Signal Processing, vol. 28, pp. 57-74, 2014.
[36] S. Zhou and G. Giannakis, "Optimal transmitter eigen-beamforming and space-time block coding based on channel correlations," IEEE Trans. Inf. Theory, vol. 49, no. 7, pp. 1673-1690, 2003.

[37] G. H. Golub and C. F. V. Loan, Matrix Computations, 3rd ed. John Hopkins University Press, 1996.

[38] Y. Yamada, S. Tazaki, and R. Gray, "Asymptotic performance of block quantizers with difference distortion measures," IEEE Trans. Inf. Theory, vol. 26, no. 1, pp. 6-14, 1980.

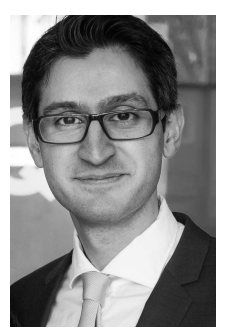

Amirpasha Shirazinia (S'08) received the BSc and MSc degrees, in communication systems, from Ferdowsi University of Mashhad, and Amirkabir University of Technology (Tehran Polytechnic), Iran, in 2005 and 2008, respectively. He received his PhD degree in Telecommunications from KTH-Royal Institute of Technology, Department of Communication Theory, Stockholm, Sweden, in 2014. Dr. Shirazinia is currently a post-doctoral researcher at Uppsala University, Sweden. His research interests include joint source-channel coding, statistical signal processing, compressed sensing and sensor networks.

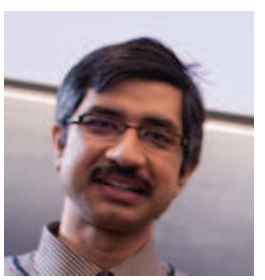

Saikat Chatterjee is a researcher jointly with the Communication Theory and the Signal Processing Divisions, School of Electrical Engineering, KTHRoyal Institute of Technology, Sweden. He was also with the Sound and Image Processing Division at the same institution as a post-doctoral fellow for one year. Before moving to Sweden, he received Ph.D. degree in 2009 from Indian Institute of Science, India. $\mathrm{He}$ was a co-author of the paper that won the best student paper award at ICASSP 2010. His current research interests are source coding, speech and audio processing, estimation and detection, sparse signal processing, compressive sensing, wireless communications and computational biology.

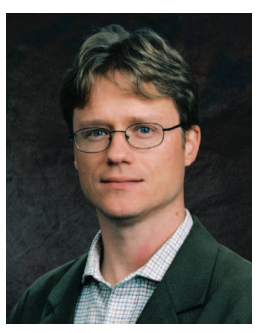

Mikael Skoglund (S'93-M'97-SM'04) received the Ph.D. degree in 1997 from Chalmers University of Technology, Sweden. In 1997, he joined the KTHRoyal Institute of Technology, Stockholm, Sweden, where he was appointed to the Chair in Communication Theory in 2003. At KTH, he heads the Communication Theory Division and he is the Assistant Dean for Electrical Engineering. He is also a founding faculty member of the ACCESS Linnaeus Center and director for the Center Graduate School.

Dr. Skoglund has worked on problems in sourcechannel coding, coding and transmission for wireless communications, Shannon theory and statistical signal processing. He has authored and co-authored more than 100 journal and 250 conference papers, and he holds six patents.

Dr. Skoglund has served on numerous technical program committees for IEEE sponsored conferences (including ISIT and ITW). During 2003-08 he was an associate editor with the IEEE Transactions on Communications and during 2008-12 he was on the editorial board for the IEEE Transactions on Information Theory. 